\title{
Mathematical Modelling of Bacterial Quorum Sensing: A Review
}

\author{
Judith Pérez-Velázquez ${ }^{1,2}$ (D) Meltem Gölgeli ${ }^{1,3}$ • \\ Rodolfo García-Contreras ${ }^{4}$
}

Received: 26 March 2015 / Accepted: 15 March 2016 / Published online: 25 August 2016

(C) Society for Mathematical Biology 2016

\begin{abstract}
Bacterial quorum sensing (QS) refers to the process of cell-to-cell bacterial communication enabled through the production and sensing of the local concentration of small molecules called autoinducers to regulate the production of gene products (e.g. enzymes or virulence factors). Through autoinducers, bacteria interact with individuals of the same species, other bacterial species, and with their host. Among QS-regulated processes mediated through autoinducers are aggregation, biofilm formation, bioluminescence, and sporulation. Autoinducers are therefore "master" regulators of bacterial lifestyles. For over 10 years, mathematical modelling of QS has sought, in parallel to experimental discoveries, to elucidate the mechanisms regulating this process. In this review, we present the progress in mathematical modelling of QS, highlighting the various theoretical approaches that have been used and discussing some of the insights that have emerged. Modelling of QS has benefited almost from the onset of the involvement of experimentalists, with many of the papers which we review, published in non-mathematical journals. This review therefore attempts to give a broad overview of the topic to the mathematical biology community, as well as the current modelling efforts and future challenges.
\end{abstract}

$凶 \quad$ Judith Pérez-Velázquez

perez-velazquez@helmholtz-muenchen.de

1 Institute of Computational Biology, Helmholtz Zentrum München, German Research Center for Environmental Health, Ingolstädter Landstr. 1, 85764 Neuherberg, Germany

2 Centre for Mathematical Science, Technical University Munich, M12 Boltzmannstr. 3, 85747 Garching, Germany

3 Department of Molecular Biology and Genetics, Bilkent University, SB2, 06800 Ankara, Turkey

4 Departamento de Microbiología y Parasitología, Facultad de Medicina, Universidad Nacional Autónoma de México, Ciudad Universitaria, Av. Universidad 3000, CP. 04510 Ciudad de México, D.F., Mexico 
Keywords Bacteria - Communication - Quorum sensing · Antibacterial · Autoinducers $\cdot$ Mathematical modelling $\cdot$ Simulations

Mathematics Subject Classification 05C38 - 15A15 - 05A15 - 15A18

\section{Introduction}

Quorum sensing (QS) is a cell-to-cell bacterial communication mechanism among the same or different bacterial species, which is enabled through small diffusible signal molecules which bacteria produce (autoinducers) and perceive (inducers). When a stimulatory threshold of signalling molecules secreted by other cells is encountered, this activates transcription of several genes (see Fig. 1). This coordinated behaviour (we later discuss whether the entire population is involved) of bacterial cells is used in a variety of forms (QS-regulated processes). Among QS-regulated processes are aggregation, luminescence, biofilm formation, and virulence factor production (see Table 1 for a review including the references of associated mathematical models).

QS is a central topic in microbiology and considered as one of the most "consequential stories" in molecular microbiology (Winzer et al. 2002; Busby and Lorenzo 2001) with hundreds of new publications every year (Web of Knowledge) and several thousand since QS was first observed in the marine bioluminescent bacteria Vibrio fischeri (Hastings and Nealson 1977; Nealson and Hastings 1979) in the late 1970s. It is also, however, a great source of debate with many publications (Hense et al. 2007; Platt and Fuqua 2010; Redfield 2002; Stacy et al. 2012; West et al. 2012) dedicated to questioning the current understanding of the process which underlays the term quorum sensing, introduced by Fuqua et al. (1994).

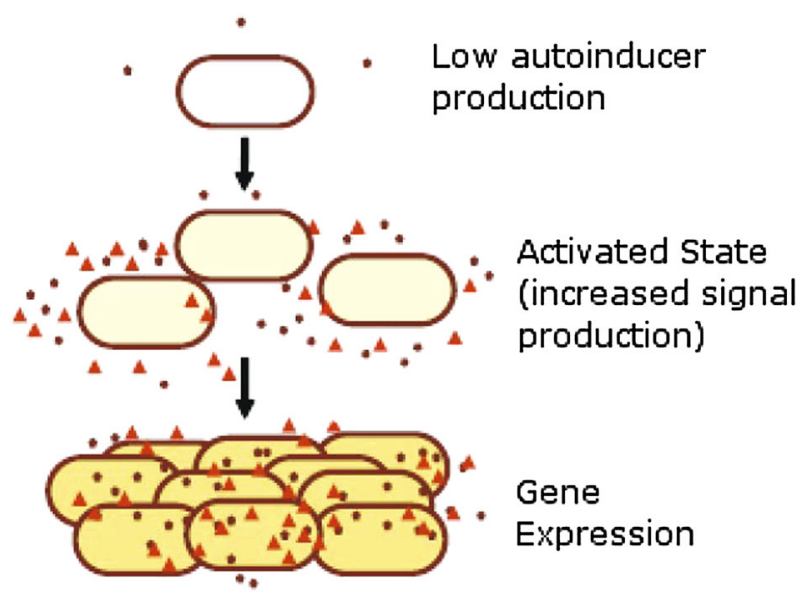

Fig. 1 At low cell density, there is a low autoinducer concentration. As the population grows, a certain autoinducer concentration threshold leads to QS activation, which in turn generates increased signal production, leading to coordinated changes in gene expression. Taken from Biology Direct 2009, 4:6 
Table 1 QS-regulated processes

\begin{tabular}{|c|c|c|c|}
\hline $\begin{array}{l}\text { QS-regulated } \\
\text { process }\end{array}$ & Bacteria & Mathematical model & QS signalling \\
\hline Antibiotics & Pseudomonas aeruginosa & Anguige et al. (2004, 2005) & \\
\hline Bioluminescence & Vibrio fischeri & $\begin{array}{l}\text { James et al. (2000), Kuttler and Hense } \\
\text { (2008), Romero-Campero and Pérez- } \\
\text { Jiménez (2008), Perez et al. (2011), } \\
\text { Müller et al. (2008) }\end{array}$ & AHL \\
\hline \multirow{3}{*}{$\begin{array}{l}\text { Biofilm formation } \\
\text { and maturation }\end{array}$} & Pseudomonas putida & Barbarossa et al. (2010) & AHL \\
\hline & $\begin{array}{l}\text { Vibrio cholerae, } \\
\text { Vibrio harveyi }\end{array}$ & Hunter et al. (2013) & AHL \\
\hline & $\begin{array}{c}\text { Pseudomonas } \\
\text { aeruginosa }\end{array}$ & Chopp et al. (2002a, b), Ward et al. (2003) & \\
\hline Competence & $\begin{array}{r}\text { Streptococcus } \\
\text { pneumoniae }\end{array}$ & Karlsson et al. (2007) & CSP \\
\hline Exopolysaccharides & - & Frederick et al. (2011) & \\
\hline $\begin{array}{l}\text { Motility (e.g. } \\
\text { swimming, } \\
\text { foraging) }\end{array}$ & $\begin{array}{l}\text { Pseudomonas } \\
\text { syringae }\end{array}$ & Pérez-Velázquez et al. (2015) & AHL \\
\hline Sporulation & - & Tang et al. (2007), van Gestel et al. (2012) & \\
\hline \multirow[t]{3}{*}{ Virulence } & $\begin{array}{c}\text { Pseudomonas } \\
\text { aeruginosa }\end{array}$ & $\begin{array}{l}\text { Dockery and Keener } \\
\text { (2001), Fagerlind et al. } \\
(2003,2005), \text { Viretta } \\
\text { and Fussenegger } \\
\text { (2004), Netotea et al. } \\
(2009)\end{array}$ & AHL, AHQ \\
\hline & $\begin{array}{l}\text { Staphylococcus } \\
\text { aureus }\end{array}$ & $\begin{array}{l}\text { Koerber et al. (2005), } \\
\text { Jabbari et al. (2010), } \\
\text { Gustafsson et al. (2004) }\end{array}$ & AIP \\
\hline & Escherichia coli & Li et al. (2006) & $\begin{array}{l}\text { AI-2, Indole } \\
\text { Lee et al. } \\
(2007)\end{array}$ \\
\hline
\end{tabular}

See Taga and Bassler (2003), Miller and Bassler (2001) and West et al. (2012) for a more comprehensive list. Note that not all models concentrate on a particular bacterium

Besides the traditional paradigm of QS, alternative models suggest it evolved not to enable bacteria to estimate cell density but to indicate whether the diffusion or mass transfer is low. Redfield (2002) suggested that QS helps determine whether secreted molecules rapidly move away from the cell. In this paradigm (called diffusion sensing), cells rely first on the secretion of metabolically inexpensive autoinducers to later safely invest in the production and secretion of the most costly molecules such as siderophores or degradation enzymes. QS is therefore employed due to its collective beneficial effects while diffusion sensing is used for individual benefits.

However, a third paradigm, efficiency sensing, unifies both concepts and solves potentially significant problems such as the negative effects of cheaters, which do 
not produce or over produce signals. Another issue efficiency sensing tackles is signalling in complex environments in which the spatial distribution of cells can be more important for sensing than cell density. Indeed, simple mathematical models show that the spatial arrangement of autoinducer producing bacteria allows cell clusters to autoinduce. In essence, the efficiency sensing paradigm states that cells measure a combination of their density, limitations to autoinducer mass transfer, and spatial distribution simultaneously and that this phenomenon evolved because it provides both individual and group fitness benefits (Hense et al. 2007).

The debate about the ecological and evolutionary function of QS coincided with the state of knowledge at the time. The first reports of QS appeared when observed only at high cell density. Nowadays, it is known that many factors besides cell density are involved in autoinduction, e.g. environmental $\mathrm{pH}$ and temperature (Uroz et al. 2005), diffusion and advection (Redfield 2002), spatial distribution of cells (Boyer and Wisniewski-Dyé 2009). That is, bacteria use QS signals to infer social (density) and physical (mass transfer) properties of the environment.

Among the most well-known QS-regulated processes are biofilm formation (Sect. 2.2.1), bioluminescence (Sect. 2.2.2) and, perhaps the most relevant to human health, virulence (Sect. 2.2.4). However, QS is also pivotal in the environment such as degradation processes in sewage plants and nitrogen cycling (Hense and Schuster 2015). Table 1 lists a few well-known QS-regulated processes.

Given that QS, or more generically, autoinduction (AI), controls a wide variety of functions, the question of whether there are unifying principles that underlie all AI systems has been explored by Hense and Schuster (2015). They argued that such core principles do exist, which is why mathematical modelling remains a powerful tool for understanding the regulation of QS. Employing mathematical tools to study QS has given rise to a wide range of mathematical models: while the autoinducer production and its binding to the regulator protein may be explained deterministically (Dockery and Keener 2001; Nilsson et al. 2001), binding of the autoinducer/regulator protein to a promoter region of DNA is often examined stochastically (Goryachev et al. 2005; Müller et al. 2008; Weber and Buceta 2013). Models involving the spatial effects of the environment, e.g. diffusion or advection, have put forward hybrid approaches (Müller et al. 2008; Vaughan et al. 2010; Hense et al. 2012). See Fig. 2). The various mathematical modelling approaches employed so far include mostly continuous (differential equations) and discrete (cellular automata or agent-based models) models, stochastic and evolutionary models. A rough classification as a summary is found in Table 3.

The most recent findings regarding QS include the discovery of a broad set of bacterial strategies such as combinatorial QS (Cornforth et al. 2014), phenotypic switch (Dumas et al. 2013), and reversible non-genetic phenotypic heterogeneity (Pradhan and Chatterjee 2014), possibly to cope with changing environments.

One of the aims of this review was to create awareness of mathematical modelling of QS in the mathematical biology community, as many of these works have been published in biology journals. To provide a broad overview, we classify the models in a series of categories involving their modelling goal, rather than the mathematics employed to address the issue, as many models involve more than one mathematical approach. Section 2.1 examines the modelling of the QS molecular mechanism; Sect. 2.2 surveys models of QS-regulated processes; Sect. 2.3 presents models that 


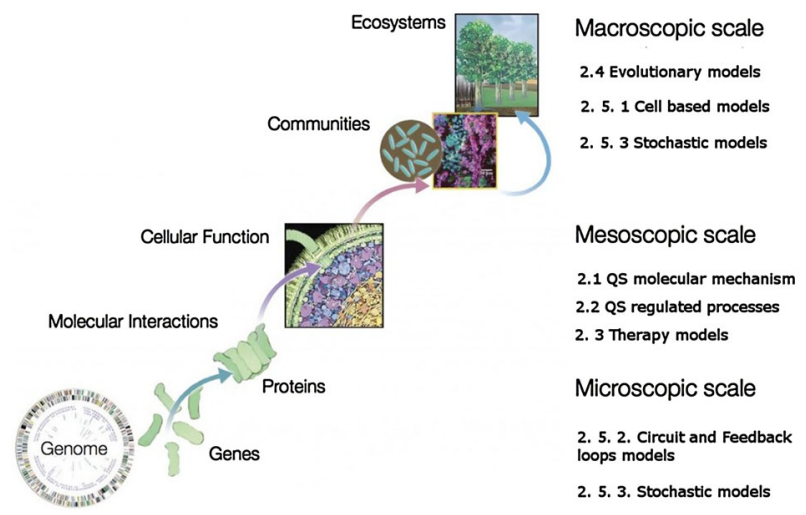

Fig. 2 Multiscale nature of QS ranges from molecular to population interactions. We indicate in which section the associated models can be found. Reproduced with permission from Dr. Tomas Perez-Acle

have investigated whether targeting QS can be used as a therapeutic strategy; Sect. 2.4 discusses evolutionary models; and finally, Sect. 2.5 describes novel approaches to understanding QS, including models of QS from a single-cell viewpoint or as a signalling circuit. Some papers fall in more than one category, and we will note its relevance when appropriate. The review is not intended to be exhaustive, but should give the reader a broad overview of the topic. Although this review is for bacterial QS, cellto-cell communication takes place in other cell types; therefore, we mention a couple of examples concerning QS of immune cells. We conclude with a discussion of future challenges in the field of mathematical modelling of QS. In Box 1, a glossary of necessary terminology is presented. Table 2 shows a timeline of research highlights in QS.

\subsection{The Signalling System}

At low cell density, the autoinducer is synthesized at basal levels and diffuses into the surrounding medium, where it is diluted. With increasing cell density, however, the intracellular concentration of the autoinducer rises until it reaches a threshold concentration beyond which it is produced autocatalytically, resulting in a dramatic increase (positive feedback). See Fig. 3.

Bacterial QS systems can be roughly divided into the two main types of bacteria: Gram negative and Gram positive (the cell wall type being the main difference).

Gram-Negative Bacteria generally use acylated homoserine lactones as autoinducers (AIs); a single QS process in Gram-negative bacteria has a gene regulatory system that includes two essential components: the inducer protein (known as I, e.g. $L u x I)$ synthesizes the autoinducer molecules and the transcriptional regulator protein (known as R, e.g. $L u x R$ ), interacts with the autoinducers molecules (e.g. AHL) and forms a complex. Bacterial growth causes AIs accumulation. AIs diffuse freely through the cell membrane or are otherwise efflux and outspread spatially. The receptor-autoinducer complex (R-AI) binds to the promoter of the protein operon on the DNA to trigger the positive feedback loop for an increasing production of AIs (Waters and Bassler 2005) (Fig. 4). 
Box 1 Glossary

Acyl homoserine lactones (AHL): Preferred QS autoinducer signals in Gram-negative bacteria Autocrine: Cells capable of producing the signal and also receiving it

Autoinducers: QS signal molecules that accelerate their own production

Biofilm: Tri-dimensional structures attached to biotic or abiotic surfaces, made of an extracellular matrix and cells in which bacteria and other micro-organisms are mostly found in nature

Competence: is the ability of a cell to take up extracellular DNA from its environment

Exopolysaccharides: Polymers which protect from environmental stresses and can be a component of biofilms

Exoproducts: Molecules exported outside cells to exert their functions, e.g. exoproteases, siderophores

Extracellular polymeric substances (EPS): are high molecular weight compounds mostly composed of polysaccharides (exopolysaccharides)

Evolutionarily stable strategy (ESS): is a strategy which, if adopted by a population in a given environment, cannot be invaded by any alternative strategy

Gram-negative bacteria: Bacteria with a double external membrane and a periplasmic space

Gram-positive bacteria: Bacteria with only one external membrane and a thick cell wall made mostly of peptidoglycan

Lactonase: Enzyme that degrades AHL autoinducers

Opportunistic pathogen: One that normally is not harmful to healthy individuals but attack immunosuppressed or compromised ones, e.g. of bacterial opportunistic pathogens: P. aeruginosa, Chromobacterium violaceum

Quorum sensing (QS): Cell-to-cell communication involving the production and detection of autoinducers that allow bacteria to coordinate gene expression as a function of cell density

Quorum quenching (QQ): The process of attenuating QS by disrupting signal production or perception

Resistance: Innate or acquired ability of a micro-organism to be impervious to the inhibitory effects of growth antimicrobials or anti-infective in virulence

Social Cheaters: Individuals that enjoy the benefits of a cooperative trait without investing in its production. Bacteria social cheaters had been identified in $P$. aeruginosa and other bacterial species, these are QS mutants that do not produce beneficial exoproducts like siderophores or exoproteases but enjoy the iron delivered by the siderophores made by the cooperative individuals or the amino acids/peptides generated by the exoproteases made by cooperative individuals

Swarming: Concerted movement of a bacterial community in a given direction, for example towards nutrients

Virulence: Parasite-induced damage to the host

Gram-Positive Bacteria use processed oligopeptides; QS in Gram-positive bacteria differs from the Gram-negative bacteria on the type of AIs (e.g autoinducing peptides) used and the perceiving mechanism of cells. Peptides are bound by receptors present in the cell membrane and do not diffuse freely through the cell membrane. Binding of the peptide to the receptors causes phosphorylation of proteins in the cytoplasm. The phosphorylation of the transcription factor promotes changes in gene expression (Waters and Bassler 2005) (Fig. 5). 
Table 2 Timeline of modelling of quorum sensing, in cyan are biological research highlights, in black mathematical research highlights, and in magenta are research findings as a result of interdisciplinary work

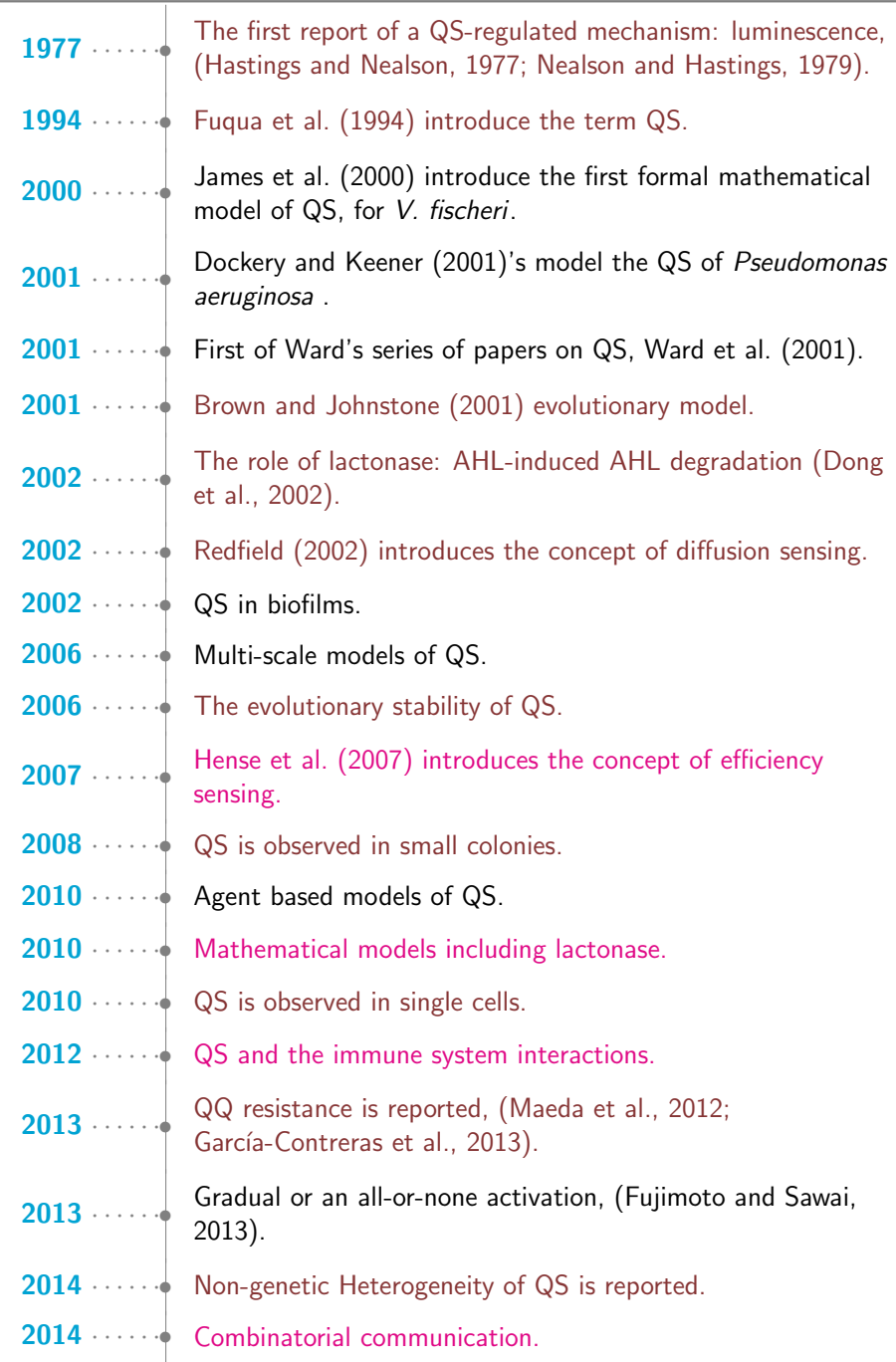

\subsection{Quorum-Sensing Molecules}

Currently, three main types of QS molecules are known:

1. Acyl homoserine lactones (AHL) mediate QS in Gram-negative bacteria, and there are several types depending on their length of acyl side chain. AHL are able to diffuse through the cell membrane. AHL are synthesized by an autoinducer synthase 


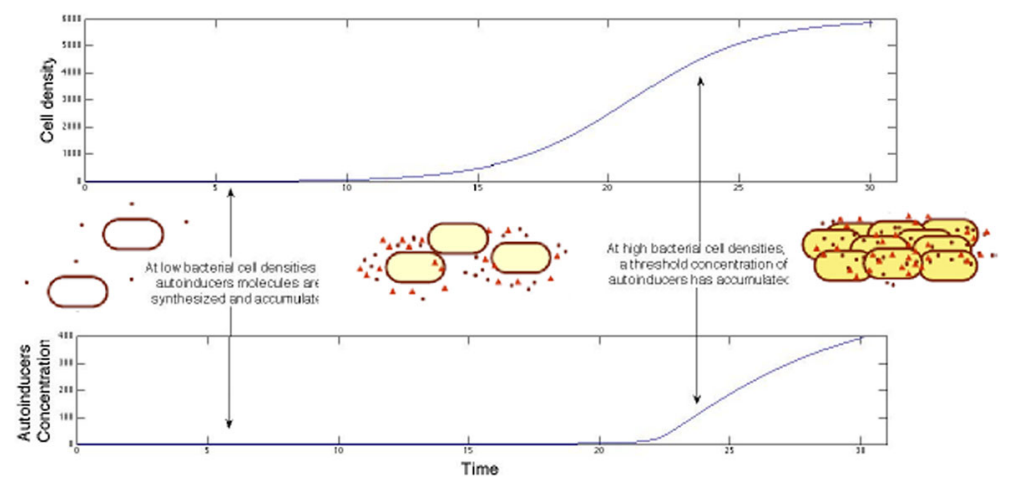

Fig. 3 How the cell density and the autoinducers concentration changes with time. Note however that QSregulated enzymes which degrade autoinducers have been reported Dong et al. (2002); therefore, strictly speaking the AHL curve is not monotone increasing, see discussion section and Fekete et al. (2010). For this particular example however, the data correspond to QS system of $P$. syringae, kindly provided by Beatriz Quinones from the US Department of Agriculture, see Pérez-Velázquez et al. (2015) for relevant details

LuxI and recognized by an autoinducer receptor/DNA-binding transcriptional activator protein $\operatorname{LuxR}$. In addition, lactonases can also degrade and inactivate AHL (Dong et al. 2002).

2. Autoinducer peptides (AIP) are small peptides that regulate gene expression in Gram-positive bacteria such as Bacillus subtilisand Staphylococcus aureus. AIP are recognized by membrane-bound histidine kinase receptors and regulate processes such as competence, sporulation, and virulence factor production.

3. Autoinducer-2 (AI-2): It is believed to be involved in interspecies communication among bacteria, as is present in both Gram (+) and Gram (-) bacteria. Chemically it is a furanosylborate diester. See Kumar et al. (2013) for more novel peptides belonging to various chemical classes.

A database of quorum-sensing peptides is available under the name Quorumpeps (Wynendaele et al. 2013), see Fig. 6 for examples of the AHL class of autoinducers.

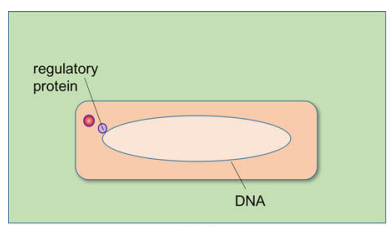

(a)

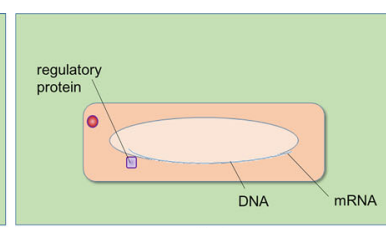

(b)

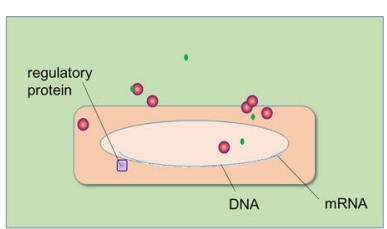

(c)

Fig. 4 In Gram-negative bacteria, the signal molecule is AHL, when the signal molecule reaches a threshold concentration $\mathbf{a}$ binds to and activates a regulatory protein which $\mathbf{b}$ binds to a specific site on the DNA $\mathbf{c}$ the binding of this regulatory protein transcription activator results in the production of the specific quorumdependent protein as well as enzymes to make more AHL 


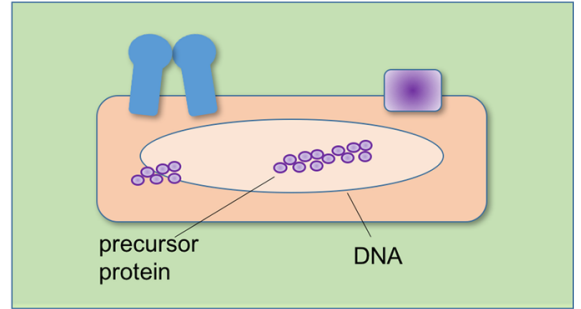

(a)

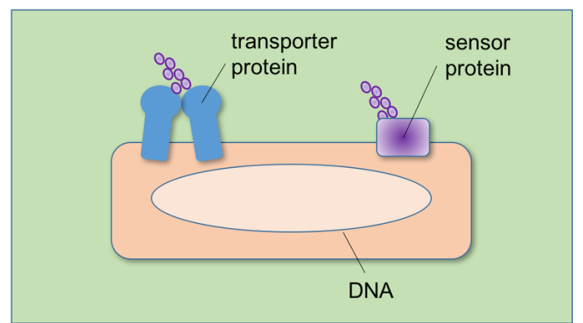

(c)

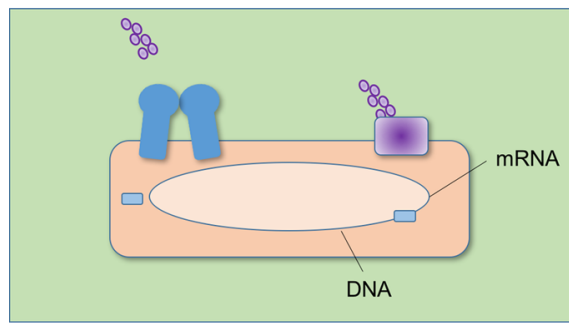

(e)

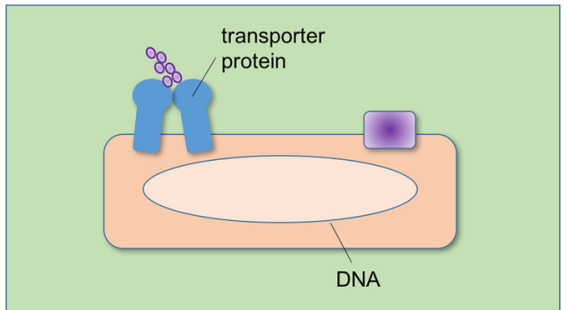

(b)

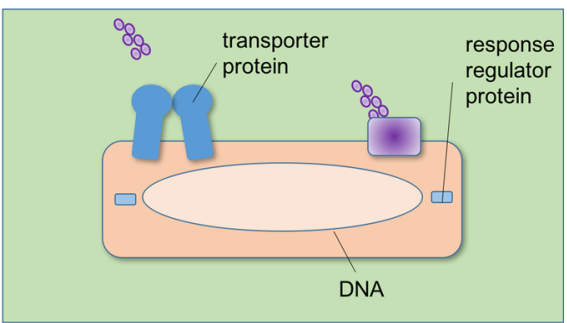

(d)

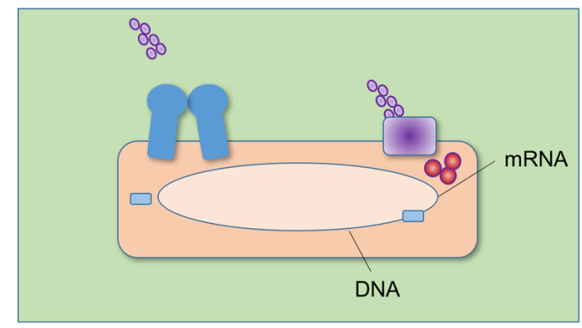

(f)

Fig. 5 QS in Gram-positive bacteria involves a different type of signal molecule; a a precursor oligopeptide is cleaved into functional signal molecules of 10 to 20 amino acids; $\mathbf{b}$ these molecules are actively transported out of the cell through a special transporter protein; c when the signal oligopeptides reach a threshold concentration on the outside the cell, they are detected by a sensor protein on the surface of the cell; d when the oligopeptide reacts with the sensor protein, the protein becomes phosphorylated on the inside the cell membrane; e the phosphate is then transferred to a response regulator protein which allows it to bind to a specific site on the DNA; $\mathbf{f}$ this binding results in alteration in the transcription of target genes, and quorum-dependent protein is produced

\section{Mathematical Modelling of Quorum Sensing}

In this review, we present mathematical models of bacterial QS, which we divide into five categories: models investigating the QS molecular mechanism, models studying specific QS-regulated processes, therapy-related models, evolutionary models, and other approaches to understanding QS (including single-cell-based models). This categorization is more in biological terms, rather than in terms of mathematical methods, given that most models use a combination of approaches. Some papers can be put in more than one category but will be dealt with the first time they appear.

Before starting the review, it should be mentioned that many computational models of QS have been proposed. A thorough review by Goryachev (2011) discussed this type of model up to 2011. Some of the models review here include computa- 


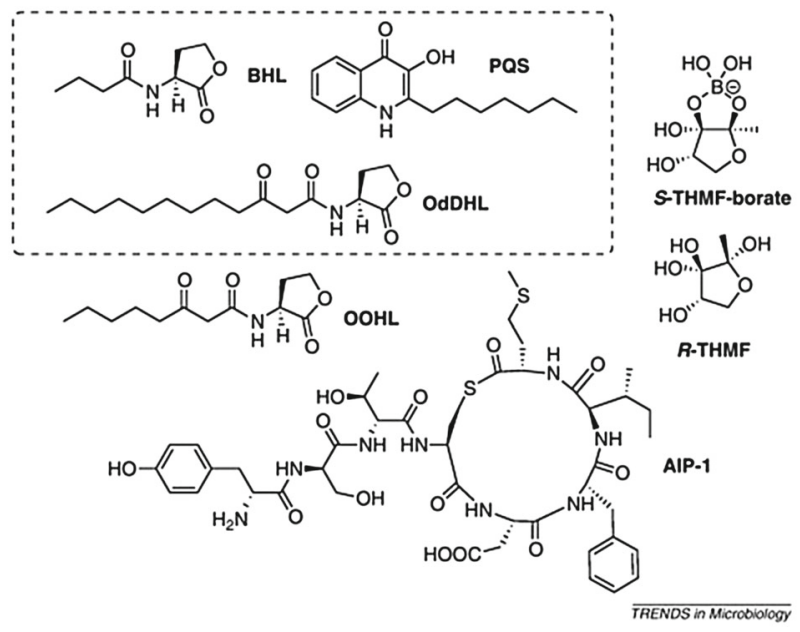

Fig. 6 BHL, OdDHL, and OOHL are examples of the AHL class of autoinducers. S-THMF-borate and R-THMF are known AI-2 signalling compounds. Picture from Galloway et al. (2012). Reproduced with permission

tional approaches; therefore, some works reviewed in Goryachev (2011) will also be examined here.

\subsection{Models of the QS Molecular Mechanism}

This category consists of models examining the generic regulation system of QS, i.e. models of the biochemistry of autoinducer regulation, which are considered to be seminal papers on the mathematical modelling of QS. As mentioned in the previous section, the regulation network includes interactions between the inducer protein and the transcriptional regulator protein with the resulting complex, triggering a positive feedback loop. More complex interactions have also been described, see Goryachev (2011, 2009) for a review of the various layouts, including single positive feedback, additional positive feedback, and negative feedback.

The mathematical modelling of QS started ${ }^{1}$ with the almost simultaneous publications of three groups: James et al. (2000), Dockery and Keener (2001), and Ward et al. (2001). The first two concentrated on the molecular mechanism, whereas Ward et al. (2001) focused on cell growth and autoinducer production. The model of James et al. (2000) was developed for the QS system of V. fischeri (Gram-negative bacteria); it took a deterministic form and focused upon the regulatory system within a single cell accounting for the cellular and extracellular concentration of AIs. The equations have two stable metabolic states corresponding to the expression of the luminescent and non-luminescent phenotypes. The corresponding first-order nonlinear ODE system has three steady states, one of which is stable and the other two are stable under certain conditions which lead to a "switch-like" behaviour of the regulation system. The

\footnotetext{
1 A mathematical framework was used before to address QS in the context of evolution, see Sect. 2.4.
} 


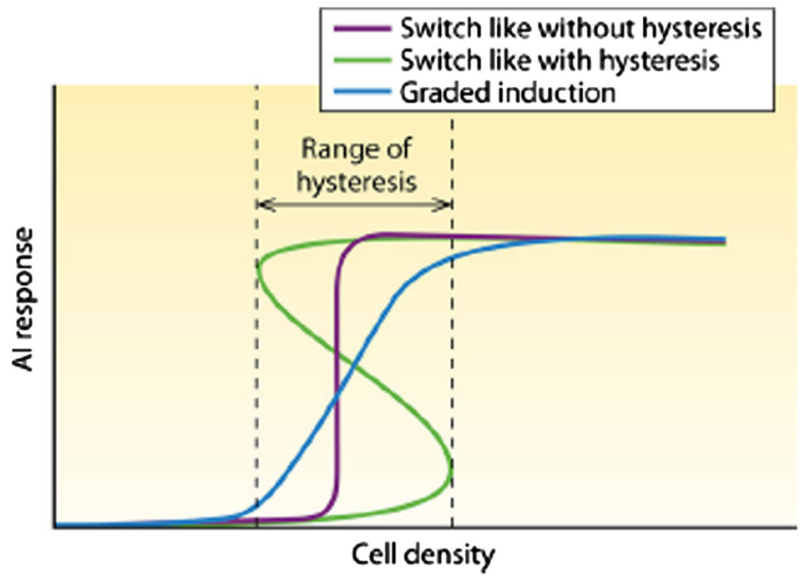

Fig. 7 Nonlinear dynamics of the activation of a generic QS (auto-inducer) system, following Hense and Schuster (2015), who argue there exist core principles in all bacterial autoinducer systems. Bistability means the existence of two stable states at the same cell density (an off state and an on state), which is often associated with hysteresis

production and loss of regulatory proteins and autoinducer molecules were examined by means of the chemical kinetics of the system.

Dockery and Keener (2001) presented one of the first mathematical models of QS. Their model examines the QS of Pseudomonas aeruginosa, a human pathogen. At that time, it was known that these bacteria possess two regulatory QS systems, called the Las and the Rhl systems. In that work, they emphasized the kinetics of the Las system and described it with an eight-dimensional ODE system, taking into account Michaelis-Menten-type expressions. They simplified this ODE system by studying different timescales of certain chemical reactions: LasR and LasI enzymes live much longer than their producers, lasR mRNA and lasI mRNA, respectively. The stability of the nonlinear ODE system, which has three steady solutions, was dependent upon the parameter of the local density of cells. The two stable states switch between one with low level and one with high levels of autoinducers (bistability, see Fig. 7). Further, they extended the model in homogeneous environment to a more realistic model by adding a spatial variable.

In a paper published nearly simultaneously, Ward et al. (2001), using a population dynamics approach, developed a model of the QS system of $V$. fischeri, which examines bacterial population growth and autoinducer production rather than the biochemical mechanism of the QS regulatory system. We place it into this category as it deals directly with the effects of up-regulation. The focus was on the population dynamics for $V$. fischeri, in view of down-regulated and up-regulated subpopulations, and their switching behaviour with increasing autoinducer production. They analysed the corresponding ODE system numerically and compared it to experimental data. This is a foundational work as experiments were specifically designed to estimate the model parameters. An important biological result of this paper was identifying that AIs production is much faster in the down-regulated than the up-regulated bacterial population. 
Fagerlind et al. (2003) developed one of the first mathematical models focusing on the two QS systems of P. aeruginosa, in particular the way in which the las/rhl system and the regulators RsaL and Vfr interact. Their model is for a single bacterium and consists of a system of eight ODEs (for the concentrations of LasR, RhlR, RsaL, OdDHL, BHL and complexes). Their system has two steady states (uninduced and induced phenotypes), regulated by the concentration of the autoinducer OdDHL, which in turn is regulated by RsaL and Vfr. Like Dockery and Keener (2001) and James et al. (2000), they found that a high concentration of the autoinducers will cause the system to exhibit mono-stability. They investigated the behaviour further by examining the LasR/OdDHL complex as a bifurcation parameter and analysed the role of RsaL as an inhibitor and Vfr as a modulator. Although their experiments showed no significant difference in either the overall growth or the total (after $24 \mathrm{~h}$ ) signal production of three strains (wild type and two vfr mutant types), it was clear that Vfr plays an important role in distinct growth stages (e.g. the Vfr mutant produces fewer signals during the early phase of growth but increases its production at later stages).

Gustafsson et al. (2004) used a mathematical model to investigate the QS of $S$. aureus, a Gram-positive bacteria, specifically to determine the role of SarA in the agr system. They further used the model to examine AIP (auto-inducing peptide) antagonists. The model consists of seven ODEs for AgrC, AgrA, SarA, and complexes. Steady states and their stability, including a bifurcation analysis (in terms of the AIP concentration), were studied which showed hysteresis [typical of QS systems, Anguige et al. (2004), Dockery and Keener (2001), Fagerlind et al. (2003), James et al. (2000)]. According to their model, inhibitory AIP delays activation of the agr system.

Goryachev et al. (2006) developed QS model consisting of two positive feedback loops designed to explain the relationship between the structural organization of intracellular networks and the observable phenotype changes. Using a standard chemical kinetic approach based on the mass-action rate law, they described the intracellular QS dynamics while the extracellular concentration of autoinducer was assumed to be a free parameter. The model was not developed for a specific bacterium; however, it can be related to the work on QS of V. fischeri (James et al. 2000) and the QS network in P. aeruginosa (Dockery and Keener 2001). Goryachev et al. (2006) pointed out the importance of the dimerization of the transcription factor and of the presence of the auxiliary positive feedback loop for the switch-like behaviour of the network. They also added molecular noise.

Li et al. (2006) studied the luxS-derived autoinducer system AI-2 of E. coli employing a stochastic mathematical model often used in quantitative molecular biology called stochastic petri dish (SPN), see Goss and Peccoud (1998). Thanks to their integrative approach they were able to unveil an alternative pathway for AI-2 synthesis. The simulations helped them to discover that the synthesis rates are glucose-dependent. Other models of the AI-2 of E. coli have been proposed (González-Barrios et al. 2009; González-Barrios and Achenie 2010), see Goryachev (2011) for a review.

Müller et al. (2008) employed an innovative approach. A low-pass filter allows signals to pass at a certain threshold frequency but attenuates those signals above the threshold. This results in smoother signals, removing short-term fluctuations and leaving the longer-term trend. Müller et al. (2008) suggested that QS works as such a filter. They proposed that this mechanism helps to overcome the intrinsic stochasticity 
underlying a system where very small numbers of molecules produced per cell seem to be sufficient to induce a response. In particular, given that cells also respond to their own signal, this type of filtering ensures that cells distinguish their own signal. They proposed a statistical model and applied it to $V$. fischeri luminescence induction data. Their model has two parts, one describing the dynamics of receptor molecules (involving $p$, the probability of a receptor molecule binding to an AHL molecule, $\kappa$ and $\sigma$ complex formation parameters, and $A$ an input experiment parameter, which stands for AHL concentration) and the second one covering transcription processes. A differential equation was used to describe the transcriptional messenger and a set of linear equations to model the different steps involved in the luminescence process. They applied their model to the experiments of Kaplan and Greenberg (1985) and found that their model fitted well in spite of the simplifications and linearization involved. They found a range of molecule concentrations the signalling system is most sensitive to, which could be associated with the low-pass filter threshold.

The study of Williams et al. (2008) is another example of the use of integrative approaches to unveil hidden molecular pathways. They showed that two interlocked feedback loops are involved in controlling the autoinducer 3-oxohexanoyl-L-homoserine lactone autoinducer regulation in $V$. fischeri. They investigated two possibilities: presence and absence of the two feedback loops. The model is a system of ODEs for the LuxR concentration and LuxR-AI complexes. Some of the model predictions were tested experimentally (Fig. 8), allowing them to exhibit hysteresis (luxI expression can assume two levels in response to externally added AIs) depending on the cell's previous exposure to AIs. This suggests that two nested feedback loops are involved in controlling the lux operon. The authors discussed the advantages of such network architectures, including how to make it more robust to withstand perturbations; giving a "memory" to cell sub-populations (which remains up-regulated even if the AI concentration falls below the activation threshold); and diversification responses, providing cells with strategies to adapt to changing environments.

As part of these models of the QS mechanism, we mention the study of Fekete et al. (2010) for two reasons: it contains quantitative information which can be used to estimate parameters such as the rate of production of the signalling molecules and threshold concentration to achieve activation, often used in mathematical models of QS but seldom computed. Secondly, because of this quantitative information, the key role of an AHL-regulated enzyme which degrades AHL was identified. Their experiments consisted of measuring AHL at different phases of bacterial growth. The mathematical model is based on Müller et al. (2006) and consists of an equation describing AHL net production (involving a Hill-type function) and one describing cell growth. The model possesses bi-stability (stable resting state and stable active) with the possibility of hysteresis. To complete the AHL circuit, they examined the role of the Ppur-AHL complexes and how AHL production depends on the complex. They further added abiotic degradation and an AHL-degrading enzyme (five ODEs in total), which was needed to reproduce the data and which was regulated through an on/off switch. Additionally, they investigated how the homoserines and the homoserine-degrading enzymes interact.

Barbarossa et al. (2010) used a delay ODE system to describe the effect of lactonases (a metalloenzyme which degrades AHLs) on QS of P. putida, a plant growth-promoting 

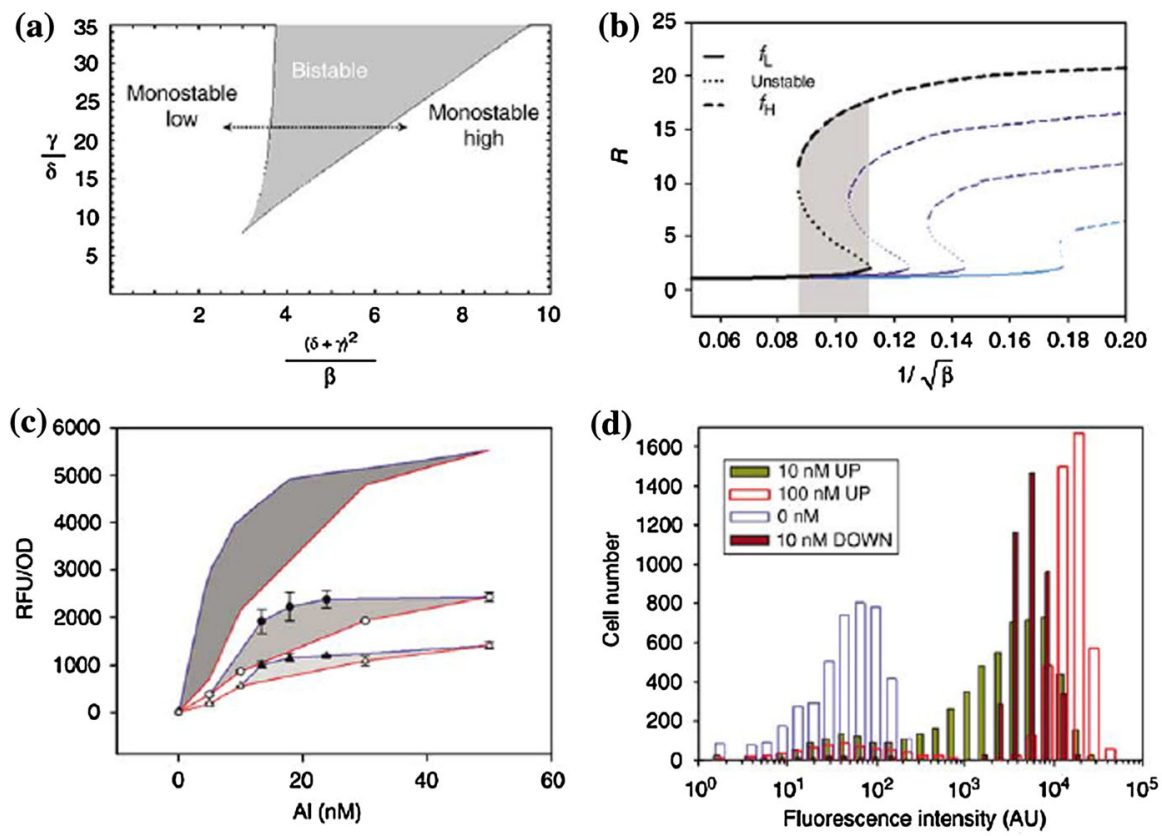

Fig. 8 Mathematical modelling and experimental analysis of the $l u x$ circuit response. a The bistability curve describing the expression of $l u x R$ as a function of [AIs]. $\mathbf{b}$ The predicted dependencies of $l u x R$ expression on [AIs]. $\mathbf{c}$ Experimental analysis of the regions of hysteresis for different glucose concentrations. $\mathbf{d}$ Flow cytometry analysis of the hysteresis in lux single-cell response. Figure from Williams et al. (2008)

bacterium which can be found on tomato plant roots. They based their model on Fekete et al. (2010) and investigated AHL dynamics. Cultures of the bacterium were grown in flasks, and measures of bacterial population density and AHL concentration were taken every hour for a period of $36 \mathrm{~h}$. The AHL concentration changes with time showed an initial maximum which was lower than a second maximum that appeared later and was followed by a steep decline (see Fig. 9). The model describes bacterial (logistic) growth, total AHL concentration, the PpuR receptor protein, AHLPpuR complex, and lactonase concentrations and has positive feedback loop. They used the experimental data to estimate growth and AHL-associated parameters. They explored the dynamics of the delay model, including bifurcation and oscillation regimes (as both positive and negative feedbacks are present). The authors chose the carrying capacity and the abiotic degradation rate as their bifurcation parameters as they can be perturbed externally. After introducing a time delay for the activation of the lactonase, the model fit the data better. This suggests that lactonase is produced and activated by bacteria only after a certain time.

Weber and Buceta (2013) used both stochastic and deterministic approaches for their QS model. Their paper explores the way in which a highly heterogeneous cell response may affect gene expression of $l u x R$. In the deterministic model, the population of cells is described by a unique volume with average and continuous concentrations of all species. In the stochastic model, cells are modelled as individual compartments and 


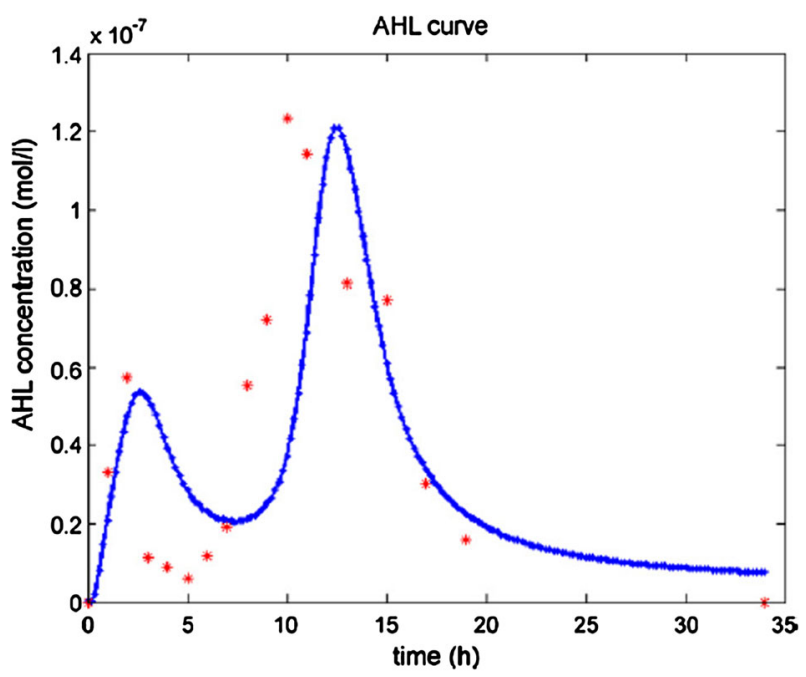

Fig. 9 AHL concentration curve, figure from Barbarossa et al. (2010). Reproduced with permission

all molecular species are represented as discrete entities. They included a noise term (on the luxR gene expression), which depends on the cell density and may influence phenotypic changes stochastically. They showed that the transition of the QS switch around the critical autoinducer concentration is very slow compared to other dynamics of the process.

Hunter et al. (2013) analysed the QS system in V. harveyi and V. cholerae, which regulates the production of virulence factors and bioluminescence, respectively. They introduced a deterministic mathematical model for a small RNA (sRNA) circuit to explain the kinetic differences and the underlying mechanisms, despite their topological and genetic similarities. The model consisted of four regulatory pathways in the sRNA circuit represented by an ODE system, which was non-dimensionalized and analysed at steady states. They fit the model to the data and found suitable parameter values for their model. Another model also exploring differences in the QS-induced luminescence phenotypes of Vibrio harveyi and Vibrio cholerae is that of Fenley et al. (2011), which extended work reported in Banik et al. (2009).

\subsubsection{QS Self-Controlling Mechanisms}

It has been argued that the general purpose of AI systems is the homoeostatic control of costly cooperative behaviours (Hense and Schuster 2015); to this end, bacteria have evolved mechanisms to repress certain components of the QS if needed. Ward et al. (2004) presented a mathematical model to investigate three of these mechanisms: the first two involve reducing the signalling molecule production (1) by a constitutively produced agent (background inhibition), and (2) due to a negative feedback process. These two processes are employed by $P$. aeruginosa. The third mechanism examines the loss of signalling molecules by binding directly to a constitutively produced agent (called soaking up), which has been observed in the plant pathogen Agrobacterium 
tumefaciens. The modelling approach consists of two sub-populations, down- and up-regulated cells, producing signal molecules at different rates. The QS signalling molecule concentration activates the switch. Their model is an extension of Ward et al. (2001). They were able to estimate some parameters using $P$. aeruginosa growth curves in liquid culture and used asymptotic analysis to explore the diverse timescales involved, which allowed them to make some simplifications in each of the three mechanisms. They also explored therapy implications by incorporating a putative drug that targets bacteria expressing a particular gene during virulence (given that their models predict that only a fraction of the population will be up-regulated if one of the suppression mechanisms is operating). Finally, they extended Ward et al. (2003) to include QS repressions in biofilm development and studied how it affects travelling wave behaviour.

The modelling of the QS regulation system has evolved significantly. Early models were mostly monomeric while recent models have tended to be of higher order. See Goryachev (2011) for a review of this particular type of models. Other models in this area are those from Mehra et al. (2008), Brown (2010), and Chen et al. (2004), which are not included here as they have already been reviewed in Goryachev (2011).

Overview and Future Research Directions The first mathematical models of QS concentrated on the architecture of the signalling pathways activating QS, in particular to understand its switch-like behaviour. Models including autoinduction threshold, the effect of bacterial density, and up- or down-regulated populations were developed. Generally, there are two different focus points in these models:

- the dynamics of substances (cellular-extracellular autoinducer concentration, induced protein concentration, lactonase activity, etc.) (Fekete et al. 2010)

- the cell response to QS (number of cells, number of activated cells) (Ward et al. 2001), including bifurcations between the steady-state solutions (Dockery and Keener 2001).

These approaches focused on the relevant regulation network at either the cellular or population level, though more recent models have involved both scales. Many analytical approaches predicted mono-stability and bistability within the network. Initially, deterministic models were used, but the potential influence of stochasticity for small numbers of certain proteins was needed. Stochastic models frequently concentrated on intra- and extracellular interactions separately, often including an analysis of the effect of potential noise on the QS network (see (Koerber et al. 2005) and Sect. 2.2.4). Furthermore, a stochastic model for a single cell, which is able to describe the probability density function for the cell to be in a down- or up-regulated state, has been proposed (see Müller et al. 2006 and Sect. 2.5.1).

We propose that new models of the QS molecular gene activation should in principle integrate deactivation too. In the same way that QS activates gene expression, under certain conditions the possibility should exist to inactivate or regulate cooperation, for example, by limiting the number of cooperating cells or the extent of cooperation. For instance, in the LuxR-type family, most members function as transcriptional activators but some function as depressors. Examples of how this may be realized include negative feedback loops, detachment or enzymes that degrade QSMs, some of which have 


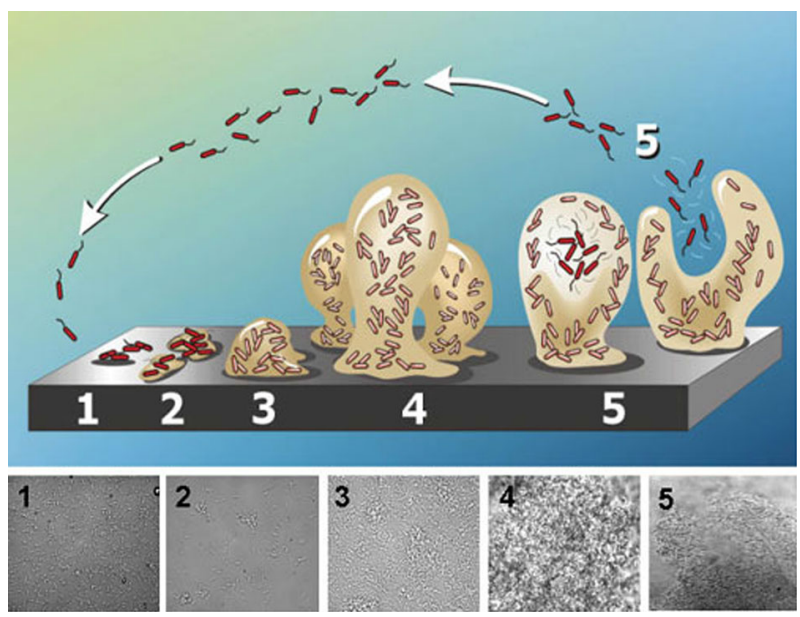

Fig. 10 Stages of biofilm development. This diagram is a cartoon of the five stages of biofilm development: initial attachment, irreversible attachment, maturation 1, maturation 2, and finally, dispersal. Under the cartoon are five electron micrographs showing what the biofilm actually looks like at each stage. Image from Monroe, D "Looking for Chinks in the Armor of Bacterial Biofilms" PLoS Biol, Vol 5, issue 11. Open access

been included in mathematical models (e.g. for the agr QS system in S. aureus which regulates detachment), but they have often not been interpreted in the context of QS as a homoeostasis regulator. See Sect. 3 for further discussion of this point.

Many mathematical models in this section assume a constant and identical AI production rate for all cells, but recent evidence of multiple AIs in one species suggests that AI production rates vary over time and independently for each QS system produced by a cell. Moreover, this is a function of the environmental and cellular conditions.

\subsection{QS-Regulated Processes}

\subsubsection{Biofilms}

Most bacteria live in biofilms (see Fig. 10), which are microbial communities attached to biotic or abiotic surfaces and encased in a matrix of extracellular polymeric substances [EPS, Costerton et al. (1999)]. This isolation protects the bacteria from antimicrobial stress in the environment. Biofilm formation is a QS-regulated mechanism, and therefore, the concentration of signal molecules is directly related to the position in the biofilm, its thickness, boundaries of the biofilm surface, etc.

Mathematical models for biofilm formation, maturation, and dissolution have been investigated for almost 30 years [see a review in, e.g. Eberl et al. (2006)]. The first models consisted of one-dimensional partial differential equations modelling a biofilm as a flat layer. Then, multidimensional models describing spatial non-uniformities were developed.

Eberl et al. (2001) developed a mathematical model of biofilm formation which is composed of a set of nonlinear density-dependent reaction-diffusion equations, which 
can be thought of as a precursor of the QS-regulated biofilm model. The model is for single-species biofilm system:

$$
\begin{aligned}
\frac{\partial C(t)}{\mathrm{d} t} & =D_{C} \Delta C-\frac{k_{1} C M}{k_{2}+C} \\
\frac{\partial M(t)}{\mathrm{d} t} & =\nabla\left(D_{M}(M) \nabla M\right)+M\left(\frac{k_{3} C}{k_{2}+C}-k_{4}\right) \\
D_{M}(M) & =d_{m} \frac{M^{b}}{(1-M)^{a}},
\end{aligned}
$$

where $D_{C}, d_{M}, k_{1}, k_{2}, k_{3}, k_{4}$ are positive constant parameters and $a>1, b>1$. $M$ denotes biomass density, and $C$ is the growth-limiting substrate. Afterwards, the dynamics affecting the spatio-temporal QS induction patterns in the developing biofilm were studied.

Nilsson et al. (2001) presented a mathematical model to describe the changes in the AHL concentration and explored the effects of biofilm growth. The model included bacterial growth using a standard logistic equation. They determined the AHL concentration within the cell and in the biofilm medium using two coupled ODEs. Solving the ODE system, they were able to track changes in AHL concentration inside the cell and in the biofilm over time and analysed the stability of equilibria graphically. They concluded that early on in population growth, high concentrations of AHL within the bacterial cell are positively affected by slow diffusion rates out of the cell and the biofilm. Therefore, bacterial growth rates impact autoinduction directly.

A more detailed model was presented by Chopp et al. (2002b) for a growing onedimensional P. aeruginosa biofilm coupled with a model of its QS systems (the las system). Their model was extended in Chopp et al. (2002a). This spatio-temporal model described the biofilm growth, oxygen, and production of the signalling molecules, where the biofilm was described in two parts: active biomass (live cells) and inactive biomass (EPS and dead cells). They studied approximate solutions to the PDE system to derive the relationship between physical parameters and signalling molecule concentration within the biofilm. Moreover, they obtained a critical biofilm depth and the corresponding approximate time for the induction of QS.

Ward et al. (2003) combined the QS activity and biofilm formation by including growth along the surface, where cell growth generates movement within the colony. They presented a nonlinear PDE model as an extension of Ward et al. (2001), with the addition of the release and diffusion of QS molecules. The model was analysed numerically, and their results agreed with the experimental data. Analytical solutions were derived by assuming uniform initial conditions. The existence of a bifurcation was investigated between a non-active and an active QS state. Finally, they investigated the travelling wave behaviour of the QS process within a certain time frame.

Janakiramen et al. (2009) developed a QS-regulated biofilm model in a closed system, namely microfluidic devices. The model comprises the mass and momentum transport in the microfluidic channel, and it explores how they impact QS and biofilm development. They showed that the flow rate has a great impact on both QS and biofilms. At higher flow rates, the stable biofilm thickness is smaller, the production 
of QS molecules reduces, and the transport rate of QS molecules out of the biofilm can be greater than the production rate, which inhibits QS. They constructed a onedimensional conservation equation for the diffusible QS molecules and combined it with a Fick's diffusion equation. The nonlinear parabolic system describes the shear stress in the microfluidic environment. The system is solved using an implicit tridiagonal finite difference scheme, and the numerical results were compared with the experimental data.

A two-dimensional model was presented by Vaughan et al. (2010) explaining the advection, diffusion, degradation, and production of signalling molecules involve in biofilm formation. They presented an finite element method to solve the reactiondiffusion-advection system and simulated the results. They concluded that for a rough biofilm surface (contrary to flat biofilm surface), less biomass may be needed to reach a quorum. Moreover, they investigated the interaction between biofilm colonies and showed that the biofilms create a region of influence where they can motivate another biofilm to reach the QS threshold.

Klapper and Dockery (2010) studied some important aspects of biofilm models including QS, growth, and antimicrobial tolerance mechanisms. They focussed on the two QS systems of $P$. aeruginosa, in particular, describing substance concentrations over time.

Frederick et al. (2011) investigated a mathematical model for QS and EPS production in a growing biofilm and analysed how a biofilm is affected by QS-regulated EPS production. The model consisted of reaction-diffusion equations, and numerical solution was computed. They concluded that low-EPS-producing biofilms generally appear in high cell populations and rapidly increase their volume to parallel high-EPS producers.

Ward and King (2012) used thin film (a thin monolayer of material) methods to address biofilm growth. The biofilm was modelled as viscous fluid. The governing equations are a system of PDEs for the biofilm volume, nutrients, two population types (up and down QS-regulated), and QS molecules. They explored two different boundary conditions between the biofilm and the sold surface: shear stress-free and no-slip conditions. For the former, they presented a hodograph solution, and for the latter, they studied the travelling wave behaviour of the resulting equation. The growth for both boundary conditions is similar, but the long-term spreading rate differs. The core of their approach was to assume that bacteria in a biofilm behave like a low Reynolds's number viscous fluid blob with volumetric growth and a small height-tolength ratio. They showed the potential of incorporating additional physical effects, in particular with regard to their effect for maturing biofilms.

Other important biofilm models are those from Kirisits et al. (2007), Duddu et al. (2009), reviewed in Goryachev (2011).

\subsubsection{Bioluminescence}

Bioluminescence (see Fig. 11) led to the discovery of QS-regulated process (Hastings and Nealson 1977; Nealson and Hastings 1979) understandingly some of the first mathematical models on QS investigate bioluminescence (James et al. 2000; Ward et al. 2001). Indeed, all mathematical models for the QS mechanism of $V$. fischeri 


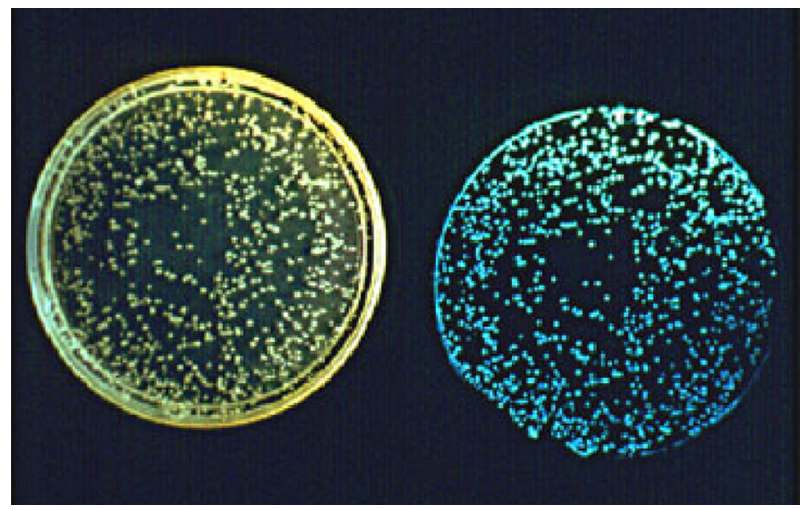

Fig. 11 Colonies of the bioluminescent marine bacterium Vibrio fischeri. This photograph of the colonies growing on agar (left) was taken with a light source. The photograph of the colonies on the right was taken using their own bioluminescence as a light source. Credit: Courtesy J. W. Hastings, Harvard University, through E. G. Ruby, University of Hawaii. Reproduced with permission

directly or indirectly deal with bioluminescence. In this section, we concentrate on the papers that specially point out this phenotypic switch in bacteria (James et al. 2000; Goryachev et al. 2006).

$V$. fischeri is a free-living marine bacterium which often appears as a symbiont of some fish and squid. At low cell densities, $V$. fischeri remains non-luminescent (see Fig. 11). V. fischeri has a QS system consisting of two main regulatory pathways (lux, involve in the production of the signalling molecule and ain in the regulation of the luminescence), Kuttler and Hense (2008) proposed an ODE system to describe the interplay between them. They extend Müller et al. (2006)'s lux model to include the ain system. Their model involves equations for external AHL concentration and the intracellular components of the regulation system. They also account for luciferase (light-producing enzyme), as luminescence (rather than AHL concentration) is most frequently measured experimentally. The number of stationary states in their system depends on external AHL concentrations. They used biochemical pathway mutants (i.e. bacterial strains missing part(s) of the full QS system) to fine-tune their model. It is known that certain strains have distinct induction behaviour (e.g. strength of luminescence, time shift) when compared to the wild type. They took ainS (no transcription of ainS is possible) and $\operatorname{luxO}$ (transcription of $\operatorname{lux} O$ is not possible) mutants. When they simplified their model to account for the first case, they obtained an lux-only like system. In the second case, they found that only one stationary state is possible. In both cases, they explored how the time course of induction becomes affected: the ain $S$ mutant starts to luminescence earlier than the wild type, and the luxO mutants shows an activation time shift.

Melke et al. (2010) proposed a cell-based QS model of growing bacterial microcolonies at the single-cell level. They analysed the molecular network with two positive feedback loops. They found regions of multistability and showed dependence of the QS mechanism on different model parameters as well on the local cell clustering. They modelled the cell response for QS as a switch from "off state" to "on state" 
and observed that the switching potential of cells is highly dependent not only on the population size, but also on the degree of local cell clustering and on the environment in which the bacteria are growing. Their model describes molecular interactions, intercellular transport, and external diffusion. Their equilibrium analysis showed that parameters can be chosen so that a single bacterium without AHL transport is in an "on" state, but close enough to the multistable region to allow inclusion of the transport terms to switch the single bacterium into its "off" state.

Majumdar et al. (2012) modelled a well-mixed population of up-regulated and down-regulated cells of $V$. fischeri and corresponding QS molecule production by a system of ODEs. They found analytical exact solutions to this complex ODE model and analysed the stability. They showed how the solutions can be interpreted in terms of the biological process.

Liu et al. (2013) presented a computational model for the switch-like regulation of the QS mechanism of $V$. harveyi that uses multiple feedbacks to precisely control signal transduction. Using the mass-action law and Michaelis-Menten kinetics, they introduced a nonlinear ODE system to include the basic principles of QS models. Their model suggests that different feedbacks play critical roles in the switch-like regulation, and more AIs cause much sharper switching. They investigated how their models relate to experimental results. As $V$. harveyi uses three signalling molecules in parallel, researchers (Tu et al. 2010; Mehta et al. 2009; Teng et al. 2010) have investigated how this bacterium seems to sense a weighted sum of the individual input signals. We refer the reader to Goryachev's review where the work of this group is discussed in depth.

Recently, a one-dimensional reaction-diffusion model for QS-regulated bioluminescence of the marine bacterium Aliivibrio fischeri was presented in Langebrake et al. (2014). The model is based on Dilanji et al. (2012) and consists of a reaction-diffusion equation for the autoinducer concentration and an equation for the LuxI concentration measured as luminescence. They showed propagating waves of activation or deactivation of the QS mechanism in a spatially extended colony and the existence of travelling wave solutions. They assumed that there exists a maximum QS signal decay rate if the cell growth rate was ignored. For QS signal decay rates less than this maximum, they found a threshold that determines whether a QS system becomes completely up-regulated or completely down-regulated. Another important result of their work is that the speed of the travelling wave of QS activation increases with the diffusion rate of the QS signal and decreases with the decay rate of the QS signal.

\subsubsection{Swarming}

Netotea et al. (2009) presented an 2D agent-based computational model for the onset of swarming in P. aeruginosa, which is controlled by threshold levels of AHL signals and secreted factors (public goods). Their experimental framework utilizes swarming agar plates that allow the growth of activated bacteria, but not of non-activated ones. They analysed the behaviour of the wild-type strain compared to two mutants: one which produces no signal, signal negative $(\mathrm{SN})$, and one that is unable to respond to the AHL signal, signal blind (SB). In their model, there is only one signal molecule, whose concentration is in equilibrium with the environment. All public goods are included 
in a generalized secreted factor $F$ which stimulate cell's metabolism and movement if a threshold concentration of $F$ is reached. The model has three states: (1) solitary or planktonic state, where cells produce low levels of signal molecule and have low rates of movement: (2) activated state, where the signal production increases and the production of secreted factor (public goods) starts and (3) swarming. During each time interval, cells move to a new location, consume nutrients, and produce AHL signals. Cells move randomly, as long as the nutrient availability suffices (i.e. they stop moving if there are not enough nutrients). Cells divide when a level of "energy" (nutrients) is reached. Activation is not "on/off" (threshold-dependent) but accounts for sensing gradients of AHL/nutrients. The model also includes diffusible materials (nutrients, AHL signals) whose concentration is bell-shaped. They assumed that producing the AHL signal is less costly than producing $F$. Their findings include the spontaneous formation of an "activation zone" (a niche where nutrients levels allow them to keep activated), which may further move towards one direction (e.g. nutrients enriched region). Although the initial conditions of their simulations are random in terms of location and metabolic states, the population eventually shows coordinated (switchlike) behaviour. Their models show how SN mutants swarm if exogenous AHL is added, but SB will not. They further used their model to explore the kinetics, which shows a typical saturation-type behaviour. They defined the "swarming fitness" as a measure of how efficiently a cell reaches a certain location in space. Higher swarming fitness was found to out-compete the less fit counterpart.

Netotea's model is able to produce dendritic growth patterns, but this was not further explored. In this regard, we refer the reader to Ben-Jacob et al. (2000), who presented a survey of patterns formed during colony development of various micro-organisms, emphasizing the pivotal role that communication plays in self-organization.

\subsubsection{Virulence}

Another QS-regulated process is virulence, observed in many species of bacterial pathogens, such as Vibrio cholerae (Zhu et al. 2002) which causes cholera; Pseudomonas aeruginosa (Lazdunski et al. 2004), a fatal pathogen for cystic fibrosis patients; and Staphylococcus aureus (Lyon and Novick 2004), known to cause infections in surgical wounds.

Staphylococcus aureus is a bacterium which causes wound infections: endocarditis and septic shock. Some $S$. aureus strains are highly resistant to antibiotics, such as the MRSA (multiresistant $S$. aureus) strain, although not all $S$. aureus strains are MSRA. It is known that the virulence factors of $S$. aureus are under QS control: $S$. aureus enters the cell and gets surrounded by a membrane, an endosome. Inside the endosome, the bacterium becomes up-regulated and destructs the membrane. Inside the cell, $S$. aureus is protected against the immune system and antibiotics. Hence, it could calmly multiply. The cell dies and the bacteria infect other cells around it. Koerber et al. (2005) developed a deterministic and a stochastic model describing the process of endosome escape of $S$. aureus focusing on the case of a single bacterium. Their deterministic model included the basis of the QS process of $S$. aureus and provided the limiting behaviour for their stochastic model. They presented a detailed asymptotic analysis for the stochastic problem supported by Monte Carlo simulations. 
Comparisons between simulations and asymptotic solutions were made. This model showed via both asymptotic and numerical solutions that the up- and down-regulation rates of the bacterium are rapid and pointed out the importance of the distribution of endosome escape times.

Karlsson et al. (2007) developed a nonlinear dynamical model to understand the regulation of the QS-regulated competence system of Streptococcus pneumoniae, which causes community-acquired infections like meningitis and bacteremia. Its QS system regulates serotype switching, evolution of virulence factors, and rapid emergence of antibiotic resistance. They focused on the molecular mechanism responsible for the abrupt shut-off of the ComABCDE system. The model's hysteretic behaviour was examined through elasticity analysis. They showed that shutdown of competence possibly occurs at the transcriptional level on the comCDE operon and showed that competence appears in waves in the mathematical model. These results are supported by experimental studies showing the appearance of successive competence cycles in pneumococcal batch cultures.

Haseltine and Arnold (2008) examined the lux circuit of $V$. fischeri and how this impacts QS operons in bacterial pathogens (e.g. bistability in the regulation of the plant pathogen Agrobacterium tumefaciens and the human pathogen $P$. aeruginosa). Their mathematical model examines three different network architectures, with a determined threshold for bistable gene expression. The models were analysed, and the steady states were shown to be a function of the population density. They showed how virulence can be "turn" on and off depending on an induction density.

Jabbari et al. (2010) studied the QS mechanism of S. aureus, an opportunistic bacteria which although form part of the human microbiome and can also cause serious diseases including pneumonia and endocartitis. This switch is QS-regulated. Their model differs from others because it focusses on the AHL concentration threshold needed to activate virulence, rather on the quorum size. QS of S. aureus is performed by the agr operon, consisting of two transcription units, making it an example of a twocomponent system (the receptor protein AgrC can detect the presence of a signal AIP and activate the response regulator AgrA). Their model follows Dockery and Keener (2001) and consists of a system of ODE representing the intracellular components of the full agr operon. Their main variable relates to the proportion of $a g r$ up-regulated cells. The system has three layers: outside the cell, the cell membrane, and inside the cell. They performed numerical simulations initially to understand the overall dynamics and used parameter values from the literature. They later use asymptotic analysis to explore the timescales involved (eight in total), in particular to understand how an up-regulated state is reached, which for this bacterium may mean becoming virulent, if sufficient signal molecule is retained in the environment of the cells. Similar to other QS systems, bistability was observed. Jabbari later extended this model and examined other QS systems.

Anand et al. (2013) proposed a computational model of the LuxI/LuxR QS system, in particular to study the effects of inhibiting QS at multiple levels: inhibit the AHL synthesis by LuxI; the degradation of AHL; the inhibition of AHL-LuxR complex formation; and the degradation of LuxR. Their ODE model is based on Goryachev et al. (2006) and accounts for the dynamics of the cell population; the intracellular protein concentrations; and the diffusible signal. They found that a combination of 
LuxI and LuxR non-competitive inhibitors may inhibit virulence. In contrast, LuxR competitive inhibitors act antagonistically with LuxI inhibitors, which may increase virulence.

Sepulchre et al. (2007) also examined virulence, it has been reviewed in Goryachev (2011).

\subsubsection{EPS Production}

Frederick et al. (2011) presented a model of QS-regulated EPS production in a growing biofilm (cells and EPS) subject to various environmental conditions. They extended a two-dimensional model of QS in patchy biofilm communities (Frederick et al. 2010) and investigated how environmental conditions such as a hydrodynamic environment and nutrient conditions affect biofilm growth and QS. They explored the hypothesis that EPS production is QS-regulated, following a series of experimental evidence. They looked at the question of how does QS affects biofilm growth and why it may be beneficial. Their model is deterministic and consists of a system of PDEs for the local density of cells and EPS (four populations: down-regulated low-EPS producers, up-regulated high-EPS producer, non-QS low-EPS producer, and non-QS high-EPS producer), EPS, nutrients, and AHL. Nutrients reach the cells by diffusion. They distinguish between up- and down-regulated cells, and regulation is controlled by AHL concentration. AHL also diffuses. To model the QS-dependent EPS production, they assume that up-regulated cells produce EPS at higher rates than down-regulated cells. With numerical simulations, they investigated the distribution of low- and high-EPS producers in the biofilm under diverse conditions (high nutrients, with and without EPS consumption, random colony placement in mixed biofilms). They found that colony growth was indeed enhanced with high-EPS-producing non-QS biofilms. They also found that the location of the colonies plays a pivotal role on EPS production induction. Moreover, they found a clear relation between biofilm composition and EPS production: at low-EPS production, the biofilm is mainly composed by cells, but at high-EPS production rates, it is mainly EPS. They discussed the advantages of using QS-induced EPS production: protection, securing nutrient supply, or out-competing other colonies (of their own or a different species).

Overview and Future Research Directions Modelling a wide variety of QS-regulated processes has led to the use of a variety of mathematical tools. While QS regulation may be understood as a reaction-diffusion problem, spatial models require the integration of fluxes, forces, and geometrical properties of the micro-environment. In terms of the analyses performed on these models, they range from asymptotic methods to reduce the complexity of a reaction-diffusion system to optimizing numerical approaches. While biofilm formation was modelled using mass and momentum transport equations, swarming was suitably described using agent-based models. Modelling of virulence often required both deterministic and stochastic approaches. Further developments in mathematical modelling of bioluminescence have appeared in parallel to novel approaches to model the basic QS molecular mechanism.

In many bacteria, QS regulates several hundred genes rather than individual phenotypes and, to add a level of complexity, not every gene may be directly connected 
with changes in the environment. This diversity has called into question whether unifying principles exist in QS systems. Hense and Schuster (2015) argue that the general purpose of AI systems is the homoeostatic control of costly cooperative behaviours.

The apparent paradox of two phenotypes possessing mutually exclusive benefits might be explained by division of labour, for example in $B$. subtilis, where both release of public goods and induction of detachment are QS-regulated. Mathematical models describing more than one QS-regulated process in the same bacteria are needed to address this interesting perspective.

Developing models which describe a QS-regulated process has shed a great deal of specific knowledge. However, the question of which processes are QS-regulated should be directly linked to the ecological and evolutionary context of a given QS system. Hense and Schuster (2015) argue that AIs regulate processes whose evolutionary benefit is strongly coupled with the efficiency of controlling environmental changes. Experimental settings such as batch cultures allow only partially to make explorations in this context, and therefore, mathematical modelling remains a powerful approach (see Sect. 2.5) to study QS.

\subsection{Therapy Models}

QS is particularly relevant to human health when it comes to pathogenic bacteria. Models concentrating in QS as a target for therapy form an important group, in particular as an alternative to antibiotics. The danger posed by growing resistance to antibiotics has been ranked along with terrorism and global warming on the list of global human threats (Davies 2011; Smith and Coast 2013). Alternatives to antibiotics therefore represent a major research challenge. Disrupting, manipulating, or targeting QS as a therapy strategy have been explored from the moment it was clear that virulence is QSregulated. To this group belong several models of QQ or other mechanisms targeting the disruption of QS, see 2.1.1 for a model that can also be placed in this section.

\subsubsection{Quorum Quenching}

Anguige et al. (2004) focused on the QS system of the human pathogen P. aeruginosa. They developed a model to investigate two strategies to disrupting QS: antagonize the transcriptional activator-AHL interaction, and destroy the AHL signal molecule. Their model is based on the works of Ward et al. (2001) and assumes a spatially homogeneous population of cells in batch culture. The cells could have one of four possible states: those with full lux boxes, those with one lux box empty and the other one full (LasRlux-box or LasI-lux-box), and those with both lux boxes empty. Their variables include concentration of the signalling molecules and complexes, extracellular concentration of 3-oxo-C12-HSL and AHL degradation enzyme concentration. The model consists of five ODEs. They explored the model behaviour when a standard antibiotic treatment is applied. They found that sufficient doses of the anti-QS agent can reduce the AHL concentration, but the treatment success depends heavily on the rest of the parameters. Anguige et al. (2005) extended this work to include well-mixed spatially structured planktonic $P$. aeruginosa populations. 
Viretta and Fussenegger (2004) developed a deterministic model of the P. aeruginosa QS network (at the transcriptional and translational levels) including the interactions between its three known QS systems las, rhl and mvfR-PQS. This model focused on understanding $P$. aeruginosa virulence and the response of QS to pharmacological interference. The QS network dynamics were described as a set of qualitative states and transitions between these states. They showed that the LasR:3-oxo-C12HSL is the network node with the highest signal integration potential (two incoming and four outgoing connections), and hence, it constitutes an optimal target for the utilization of QS inhibitors. They also proposed that degradation of the HSL signals can be an effective strategy in the initial phases, but it fails when the QS mechanism is in full operation. They validated the interference of the PQS signalling system as a useful anti-virulence target.

Fagerlind et al. (2005) presented one of the first models of QQ. Their model is for the QS molecule antagonist (which they called QS blockers, QSB) of one of the $P$. aeruginosa signalling molecules, 30-C12-HSL. Given that certain experimental evidence had shown that the sole use of an antagonist may not be as effective to inhibit QS, they explored how to further alter this QSB to improve it. They extended Fagerlind et al. (2003)'s model by introducing different QSBs with: (1) different affinity values with the R-proteins and (2) different rates of 3O-C12-HS-induced degradation of the R-proteins. They assumed that the QSBs bind to but do not activate R-proteins. Their model of eight ODEs includes the dynamics of the QSBs by assuming that they are able to diffuse through the cell membrane and bind to both LasR and RhlR, forming new complexes. To investigate the effect of adding QSBs, they consider a high stable steady state (which was shown to exist in (Fagerlind et al. 2003)), i.e. growing a colony until the cell density is high enough to induce QS. Then, they added the QSBs and asses their ability to bring this stable state down. They did this for different antagonists differing in their ability to induce degradation of the R-proteins and their affinity for the R-proteins. Their model suggests that QSB-induced degradation of LasR is key for successful QQ. They further discuss ways to increase this effectiveness, such as developing QSBs with high affinity and high degradation of LasR.

Ward (2008) presented a continuous models of QQ (anti-quorum-sensing treatments) in both batch cultures and biofilms. The biofilm is viewed as a multiphase fluid whose growth is governed by nutrients that diffuse from the surrounding fluid. They extended Anguige's series of papers (Anguige et al. 2004, 2005, 2006) on QS inhibitors, which are based on Ward et al. (2001, 2004). The model is for the LasRI QS System of P. aeruginosa. For the batch culture case, the population consists of two populations (down-regulated and up-regulated cells). The regulation is mediated by QS, whose mechanism is modelled through a series of ODEs for the concentration of LasR, LasI, AHL, and LasR-AHL complex. Through scaling, they describe the QS dynamics with one equation for the change in AHL concentration. They studied three QSI therapies: anti-LuxR agents, anti AHL agents, and anti LuxR agents. The model involves equations for the two population types, AHL, and for each of the concentration of these three agents. For the biofilm case, they viewed the bacterial subpopulations as volume fractions of the whole of living cells. The remaining space is occupied by EPS and water. Biofilm growth is assumed to be QS- and nutrient-regulated. The QS process is modelled in the same way as in the batch culture. AHL, QSIs, and nutrients 
are assumed to diffuse. Their model is one-dimensional and consists of 10 PDEs and one ODE, with growth occurring perpendicular to the a solid surface.

Ward (2008) investigated what happens when the three QSIs are administrated (by drip-feeding or by pretreatment of the growth media) and they compare their potency. For both, batch cultures and biofilms, they found that the time of application of the QSI may be a key parameter for the efficacy of the treatment. In particular, early application will delay or prevent up-regulation. However, the amount required to suppress QS increases exponentially. In biofilms, the QSI concentration needed for successful activation repression seems to be very dependent on local growth conditions. Their third QSI method (putative anti-LasI treatment) seems the most potent.

Beckmann et al. (2012) studied an evolution model of QS in populations of digital organisms, a type of self-replicating computer program, and investigated the effects of impairing QS on these populations. They used the computational evolutionary biology platform Avida (Ofria and Wilke (2004)). Their setting contemplated individual organisms (with a list of instructions, its genome) living on two-dimensional spaces (batch) consisting of cells (each cell contains an organism). The genome of the organisms contains information to create off spring (replicate). Organism communicates to each other by sending messages. Their model evolves QS (i.e. the population evolves a strategy that collectively suppresses growth if a population threshold is reached). Then, they added mutant organisms incapable of either producing or detecting the signal molecule. They found that receiver-impaired mutants prevent QS more effectively than sending-impaired mutants. They argued that this may be related to the cost of producing a signal. They further explored how, in spite of the introduction of mutants, QS is still observable (resistance). Higher resistance to receiver-impaired mutants was observed. Finally, they tested what happens when the introduction of the mutants was gradual. In this case, both mutants seem to produce equivalent levels of resistance, meaning that as the environment becomes adverse, adaptation kicks in. Although they observed "resistance", they did not validated their data experimentally. However, simultaneously, a group provided evidence that resistance to QQ is indeed possible (Maeda et al. 2012; García-Contreras et al. 2013).

Anand et al. (2013) used an experimentally validated computational model of LuxI/LuxR QS to study the effects of using inhibitors individually and in combination.

Recently, Schaadt and collaborators developed a combined model of the regulatory and metabolic network for the three QS systems of P. aeruginosa, which enabled them to investigate the effects of gene knockouts, enzyme inhibitors, and receptor antagonists. The authors focused on the PQS production and the biosynthesis of pyocyanin, an important virulence factor, and according to their model, inhibiting the enzymes that produce PQS is an ideal strategy to block the HHQ and PQS formation, but blocking the receptor PqsR that regulates its biosynthesis is more effective to decrease the pyocyanin levels (Schaadt et al. 2013).

The QQ model of González-Barrios et al. (2009) was reviewed in Goryachev (2011).

\subsubsection{Quorum-Sensing Manipulation}

Koerber et al. (2002) presented a deterministic model of PDEs for one of the QS systems of $P$. aeruginosa (3-oxo-C12-HSL) based on Ward et al. (2001). The model 
accounts for populations of down- and up-regulated cells. Parameters of their model were taken from previous work of this group. The main contribution lies on the fact that the model addresses the wound environment explicitly and how QS plays a role. They represented a wound as a two-dimensional region with layers (outer surface, uncolonized wound, bacterial layer, undamaged skin). They investigated, through numerical simulations, the evolution of the autoinducers concentration and the fraction of upand down-regulated cells under diverse wound colonization possibilities (by changing the thickness of the regions of the wound, diffusive ratio, a fully colonized wound). They put forward the idea of exploring QS disruption as a possible therapy by turning their attention to the effect of the QSM degradation term.

Anguige et al. (2006) used a multiphase approach to model biofilm formation of $P$. aeruginosa. It is an extension of Anguige et al. (2005) to include EPS production control by QS, this generates a watery ECM surrounding the bacteria. Their model incorporates four phases: live cells, dead cells, EPS, and extracellular watery fluid. Nutrients and antibiotics diffuse into the biofilm from its upper surface. They obtained a system of PDEs for the volume fractions and velocities of these phases. AHL is produced by the cells and also diffuses. EPS production depends on the level of LasR/AHL dimmer. They used numerical simulation to explore the role of QS in EPS production. They also investigate how antibiotics and QQ treatments affect biofilm formation. They found that most of the biomass in a wild-type biofilm consists of EPS and that living bacteria are agglomerated in a nutrient-rich region near the surface. Mutant biofilms (LasI-negative) are thinner and enable bacteria to uniformly distribute. Therapy seems most effective in wild-type biofilms.

Brown et al. (2009) suggested the use of the ability of QS cheats to invade cooperative, wild-type populations as a potential new strategy for treating bacterial disease. They presented ecological models of within-host competition in three cases: (a) cooperative social traits, such as the production of exoproducts; (b) spatially structured populations, which will reduce the ability of non-cooperative cheats to spread; and (c) harmful, or spiteful, social traits (anti-competitor chemicals, bacteriocins, and temperate bacteriophage viruses). The mixtures of social cheaters and cooperative individuals in $P$. aeruginosa mice infections indeed showed a reduced virulence Rumbaugh et al. (2009); however, further experiments are needed in order to test the effects of applying cheaters in previously established infections by cooperative individuals.

Other groups looking at degradation of autoinducer are Chen et al. (2005), reviewed in Goryachev (2011).

Overview and Future Research Directions Although many of the therapy models presented are theoretical, experimental evidence validates some of the central insights derived from those models. Important predictions made by the models remain to be tested and eventually exploited to develop anti-quorum therapies. The focus should be on the generation of robust anti-quorum therapies, with an emphasis on the interplay between therapy models and experimentation.

Koerber et al. (2002) developed one of the earliest models to explore the possible application of QQ as a therapeutic alternative. Importantly, this model was developed explicitly for $P$. aeruginosa infection in wounds, and to the best of our knowledge, this 
is one of the few QS therapeutic models that is not generic for bacterial infections but indeed tries to represent a specific kind of infection. Remarkably, although the model incorporates several simplifications such as modelling only the LasR QS system, it provides valuable insights, including the prediction that a combination of antibiotics that will decrease the carrying capacity of the system (number of viable bacteria) together with the degradation of the 3-oxo-C12-HSL autoinducer will synergistically reduce the expression of QS-dependent virulence factors.

Other models had found no significant enhancement of antibacterial treatments in combination with antibiotics and QS inhibitors (Anguige et al. 2004); however, Gupta et al. (2015) have demonstrated experimentally that a combination of lactonase (which degrades the HSL autoinducers) and the antibiotic ciprofloxacin could prevent the death of infected thermally injured mice, significantly reducing systemic bacterial dissemination, the severity of histopathologic lesions and promoting skin regeneration. This approach contrasts to the independent utilization of the compounds which had more modest beneficial effects, hence confirming Koerber and co-workers prediction.

The successful prediction made by some of these models should encourage the development of new specific infection models that aim to design robust anti-quorum therapies. A recent model of a quorum-mediated infection containing a far more detailed description of the complex QS systems of $P$. aeruginosa also highlights the importance of autoinducer degradation. The authors concluded that although interfering with 3-oxo-C12-HSL autoinducer, mediated QS could be useful in the prevention of bacterial colonization and the establishment of infections, once the signal concentration reaches the threshold level (i.e. activating the QS phenotypes). Removing 3-oxo-C12-HSL autoinducer will not suffice to eliminate the expression of QS-dependent virulence factors since the RhlR module will remain functional (Viretta and Fussenegger 2004); however, this important prediction has not yet been experimentally evaluated. This model also indicates that high PQS concentrations are sufficient to up-regulate rhlR independent of LasR, hence identifying PQS as a possible target for pharmaceutical treatment of $P$. aeruginosa. Experimental evidence confirms that the RhlR system does indeed have high relevance for $P$. aeruginosa virulence in vivo, at least in animal model infections (O'Loughlin et al. 2013). Furthermore, Palmer et al. (2007) show that growth in sputum promotes the synthesis of high PQS concentration, which indicates that this system may be primordial for P. aeruginosa QS-regulated virulence in the lungs of cystic fibrosis patients.

Interfering with PQS decreases the in vitro and in vivo P. aeruginosa virulence (Calfee et al. 2001; Lesic et al. 2007). The model from Schaadt et al. (2013) confirms that inhibiting the PqsR receptor is an effective strategy to inhibit the synthesis of the virulence factors pyocyanin. Since it is estimated that at least $65 \%$ of chronic infections are produced by biofilms and given that biofilm formation (particularly its maturation involving the synthesis of several matrix biofilm components such as EPS or extracellular DNA) is regulated by QS, therapy models explicitly developed to explore the effects of QS interference on biofilm infections are especially relevant. Anguiges work represents an influential contribution in this context: by modelling early-stage $P$. aeruginosa biofilm subject to treatment with topically applied anti-QS drugs and conventional antibiotics, they found that if the biofilm formation is a slow process, both kinds of anti-QS drugs are able to maintain low autoinducer concentrations. 
Importantly, for an administrated surface drug concentration, a critical biofilm depth enables treatment success; however, once this depth is reached, the treatment fails. The critical concentration of each drug increases exponentially with the biofilm thickness; hence, targeting biofilms will remain an extremely challenging task. By incorporating biofilm maturation via QS-controlled EPS production, they further predicted that the combination of anti-QS and antibiotics is the best strategy to maximize the chances of efficiently eradicating biofilm infections (Anguige et al. 2004); however, suitable experiments to address the effects of the combination of anti-QS compounds and antibiotics to test the model's predictions are still needed.

\subsection{Evolutionary Models}

The topic of how QS has evolved and, more importantly, is stable (i. e. it is a strategy which has not been out-competed) is a fundamental research topic within bacterial communication. In principle, given that the synthesis of exoproducts coordinated by quorum sensing is a cooperative behaviour, is subject to a known emerging feature: the appearance of "cheaters" mutants. These mutants contribute less (or not at all) to the effort of cooperation but still benefit from it (Popat et al. 2012). Theory predicts that, given that these cells possess a competitive advantage over the rest, the cheater population will take over, far from what is actually observed experimentally. The ecological and evolutionary function of QS is at the core of this apparent paradox. There exists a number of theories attempting to explain this paradox, which can roughly be divided into:

Direct and Indirect Individual Fitness Individuals gain fitness through the impact of their own reproduction (direct fitness) and through their impact of the reproduction of related individuals (indirect fitness) (Hamilton 1964; West et al. 2007).

Group Selection Cooperation is favoured when the response to between-group selection is greater than the response to within-group selection (Fletcher and Doebeli 2009; Queller 1992).

Further strategies have been reported to explain the population equilibrium towards a higher survival of the cooperative individuals, restricting the proliferation of potential social cheaters: "cheater punishment" (Friman et al. 2013), a strategy which appears to favours cooperation, potentially leading to lower cheat frequency; protist predators (for $P$. aeruginosa) which preferentially predate QS-deficient mutants Friman et al. (2013); higher susceptibility to abiotic stress of the QS mutants García-Contreras et al. (2015) or to the stress produced by the QS-proficient individuals (by the generation of the HCN toxic compound) (Wang et al. 2015).

Damore and Gore (2012) reviewed standard evolutionary theory techniques used to understand microbial cooperation. In the context of evolution of bacterial QS, Brown (1999) presented the first mathematical model to study the evolutionary stability of host manipulation by parasites, which critically depends on cooperation, bacterial QS being a particular case. His model includes a fitness function, modulated by relatedness. He investigates the evolutionary stable strategies (EES) using game theory. He separates between individual $I$ and group fitness $G$ functions, a decreasing and an increasing 
function, respectively. Cooperation is then costly for individuals but benefits the group. He then tests two types of group fitness. In both cases, the model predicts a group size threshold, below which manipulation (cooperation) cannot take place.

Brown and Johnstone (2001) is one of earliest mathematical models of QS, and in terms of modelling approach, it precedes that of Brown (1999). They developed a twotrait model describing the optimal level of cooperation (production of group beneficial traits) and signalling as a function of relatedness, costs to the individual, benefits to the group, and demographic parameters. Their independent variables include: $s$ an individual's investment in the production of the signalling molecule, $t$ the total concentration of the signalling molecules which it experiences, $n$ colony size, and $r$ relation coefficient. They further assumed that the individual investment in cooperation $m$ is a function of $t$. The model comprises a PDE for the fitness of an individual, this equation involves individual and group fitness functions. Although individual investments in both signalling and cooperation negatively impact individual fitness, the later positively impacts group fitness. Comparable to Brown (1999), there is a stable level of cooperation, which can be used to find stable signalling. They investigated the effect that group size distribution has on cooperative and signalling effort.

Brown and Johnstone (2001) and Brookfield (1998) have analysed group selection models, where selection at the individual level operates against cooperation, while selection at the group level favours QS.

Nadell et al. (2008) used an agent-based model of biofilm formation to explore QSregulated EPS production. They investigated why, in Vibrio cholerae, QS seems to upand down-regulate polymer secretion at high cell density. Their modelling framework is similar to Picioreanu et al. (2004) and includes colonizing cells attached to a flat surface growing in a two-dimensional space. Cells consume nutrients and produce additional biomass. They describe four strains interacting and differing in their ability to produce EPS: (1) no polymer secretion and no QS; (2) polymer secretion but no QS; (3) polymer secretion under negative QS control; and (4) polymer secretion under positive QS control. Their results suggest that QS enables bacteria to control (switch on or off) polymer secretion to increase their competitive ability against other strains or other species within the biofilm, that is, selectively synthesize EPS. This does contrast with the common view that EPS is only a public good to help binding the biofilm. Here, we take the opportunity to highlight the work at Foster's Lab on social evolution.

Czárán and Hoekstra (2009) used a stochastic cellular automata model to show that QS can evolve from initially non-cooperating strains, by selection of specific mutations as long as spatial mixing in the population is low. Cheating and exploitation were found to develop in their simulations, but although social cheaters emerge and reach substantial equilibrium frequencies, they are incapable of completely eradicate cooperators.

Overview and Future Research Directions Several studies have found that the presence of proficient QS systems is important for bacterial virulence and that in spite of putative QS cheaters, putative cooperators are very common (Castillo-Juarez et al. 2015). Interestingly, at least in the clinical setting, . a aeruginosa las $R$ mutants that can behave as social cheaters are often found; however, there are few studies that address whether these kinds of mutants can coexist with cooperative QS-proficient strains. 
Remarkably, one recent study, made with eight cystic fibrosis (CF) patients, demonstrates that each patient carries a complex mixture of QS-proficient and QS-deficient isolates. However, the study did not find a single patient with $100 \%$ QS-deficient isolates (Wilder et al. 2009), a result which supports the prediction of Czárán and Hoekstra (2009).

There is strong evidence supporting (spatial) assortment as a process to evolutionary stabilize cooperation. This could mean new colony formation, repeated cycles of colony dispersal, mixing, and regrowth. Models dealing with these issues have been put forward.

A comprehensive list of environmental factors that could in principle select the presence of functional QS systems during evolution from biotic, abiotic stress to spatial organization is provided in García-Contreras et al. (2015). Novel experiments regarding the evolution of bacterial QS systems in the laboratory are feasible, and such experimental approaches in combination with mathematical modelling can provide insights into the roles of direct and indirect individual fitness, group selection, kin selection, social cheating, and environmental variables in the rise and selection of QS bacterial systems. These evolutionary insights can provide a better understanding of QS bacterial systems.

The evolutionary stability of QS depends heavily on the environment where it takes place. Luminescence for instance seems beneficial only if produced by many cells; however, it has been speculated that oxygen detoxification is the original purpose of bioluminescence. In this view, isolated or non-cooperator cells may also benefit from activation. New experimental set-ups such as beads or dialysis bags represent an important source of new data in this respect.

The evolutionary context is of particular relevance when targeting QS as a treatment strategy. Inhibiting mechanisms may affect QS-proficient and QS-deficient bacteria differentially, depending on habitat conditions. Models investigating the long-term effect of changing environments, such as stochastic evolutionary models, should be further developed.

van Gestel et al. (2012) suggested that cell-to-cell communication evolves because cells are capable of anticipating environmental stress, which leads to ecological diversification. See Sect. 2.5.1 for more details. Here, we note the work of Beckmann et al. (2012) (Sect. 2.3.1), as it relates to evolution of QS too.

\subsection{Novel Approaches}

\subsubsection{Cell-Based Models}

Modelling QS on a population scale has been possible through the use of agent-based models, which make it possible to describe a range of conditions for individuals, for example undergoing activation at varying concentrations of autoinducers.

Müller et al. (2006) proposed a spatial single-cell model of QS. Their model describes two layers: within and between cells. It describes a population of single cells, with the QS signalling system modelled by nonlinear ordinary differential equations, containing variables describing AHL outside of the cell and within the cytoplasm. The 


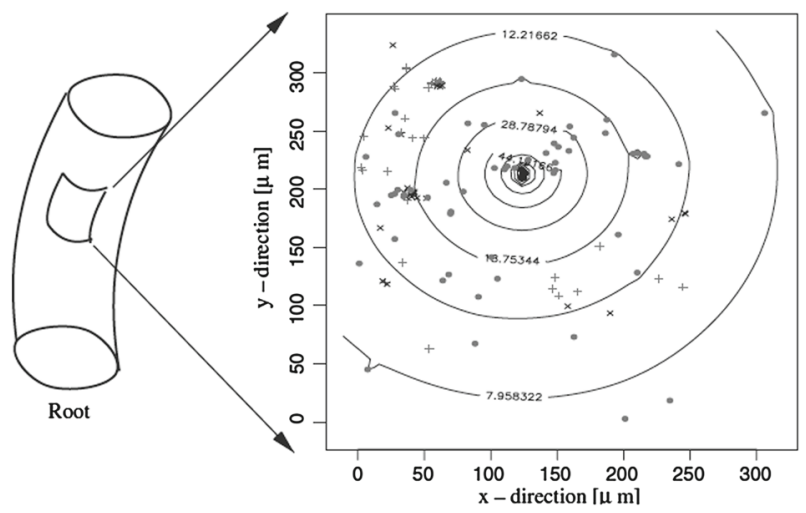

Fig. 12 Müller et al. (2006) spatial model. Circles (black if activated, grey for resting producer cells) denote the measured location of producers. Plus signs mark the measured location of resting, cross signs that of active detector cells. The equipotential lines denote the density of AHL concentration predicted by the model (normalized units). Reproduced with permission

model contains a parameter to account for the external signalling substance, effectively enabling them determine whether a cell is resting, activated, or in the bistable regime. They combined the ODE model with a PDE to give spatial structure. They assumed no spatial structure within the cell; therefore, only the influx/efflux of AHL through the cell membrane impacts the dynamics. The linear PDE describes what happens to the signalling substance outside the cells. They coupled the ODEs and the PDE via mixed boundary conditions at the cell surface. The system typically starts subcritical (low levels of the signalling substance); later, the population density grows until the critical density is reached, which leads to a jump to the activated state. Their results indicate that trajectories starting from very small or from very large initial conditions tend to stationary states. Given that the cells are quite small compared to their distance, through rescaling they obtained an approximative equation, where cells are represented as inhomogeneities on the right-hand side of the partial differential equation. They also showed how their work could be applied to spatial data obtained by CLSM for P. putida colonizing wheat roots (see Fig. 12).

Tang et al. (2007) used an individual-based model to study bacterial foraging behaviour. It is an extension of a "varying environment bacterial modelling" (VEBAM) approach (see Tang et al. 2006) to include QS. Their modelling framework is composed of two parts: the cells and the environment. The environment is a two-dimensional surface (segmented equally into small niches) used for food searching, it is defined by its characteristics such as boundary and the map of food distribution. The cells have both a receptor (to detect the value of its surrounding environment) and a decisionmaking system (which feeds from the information obtained by the receptors). They modelled QS through clusters (aggregation of population) defined by population density, position, and an autoinducer diffusion parameter. Depending on the population density, the cluster is in an "on" or "of" state. They also included the energy that cells use to produce autoinducers. Their model displays common features of QS such as that, at a low cell density, the autoinducer is synthesized at basal levels and dif- 
fuses into the surrounding medium, where it is diluted. They found that QS impacts growth and nutrient consumption: a simulation of a QS population will produces less (number) bacteria than the non-QS case. Also, a QS population achieves a higher population level with less nutrient consumed as compared to the population without QS.

A P system is a modelling framework which consists of a hierarchical arrangement of "membranes" corresponding to regions inside the system. These regions have objects and a finite set of rules of interaction. $\mathrm{P}$ systems were introduced in PérezJiménez and Romero-Campero (2006) and are based on Gillespie (1977)'s stochastic simulation of coupled chemical reactions. It can be thought of as an agent-based model, where a P system is an individual agent. Romero-Campero and Pérez-Jiménez (2008) presented a model for the QS of $V$. fischeri using this modelling framework. They examined the importance of the level of noise in the system. Their simulations showed that at low cell densities, bacteria remain dark while at high cell densities some bacteria start to be bright. They further studied how QS functions in colonies. Their results suggested that very limited information of the local environment can help coordinate collective behaviour of multiple agents.

van Gestel et al. (2012) presented an agent-based model to describe how cell communication plays a role in the sporulation of the bacterium Bacillus subtilis. They assumed cells scattered throughout the soil in locations where nutrients are available. Cells can grow and differentiate (into spores, communicating cells, or remain undifferentiated) and will enter starvation if nutrients do not suffice. Sporulation takes place if both nutrients are enough and QS signal is above a threshold. Cells can also migrate to find better locations. Communicating cells produce signalling molecule at a fixed rate, signal is also degraded. The amount of signal changes over time depending on the number of signalling cells. Sporulation is assumed to be an irreversible state which requires a specific amount of time to be completed. Spores cannot longer divide. Both division and differentiation depend on the environment (nutrients for the first and both nutrients and signal for the later). Mutations are also permitted. The time of sporulation is key; if a cell sporulates too early, it loses reproductive potential; and if it does it too late, it has more chances of dying due to the lack of nutrients. They examined how QS evolves under two scenarios: colonies are initiated by a single individual (i.e. genetic variation can only arise via mutations) or assuming within-colony variation. They further explored how signal production cost may impact the stability of QS development. They found that QS development is highly dependent on sporulation time. QS evolution depends on both signal production evolution (by differentiation) and signal sensitivity. Their model shows how QS can evolve as a mechanism to evaluate environmental cues such as nutrients availability. They further showed that QS can also evolve if there are genetic variations within the colony and under certain levels of signal production costs. See Fig. 13.

Fozard et al. (2012) used an agent-based model of a developing biofilm subjected to QQ. It represents a 3D biofilm reactor with two compartments, the biofilm compartment and the bulk liquid compartment based on Picioreanu et al. (2004) but including EPS production explicitly; a QS model (requiring particles to keep track of the number and states - up- or down-regulated-of the cells) and biomass spreading. There are three diffusible substances in the bulk liquid compartment: nutrients, QS mole- 

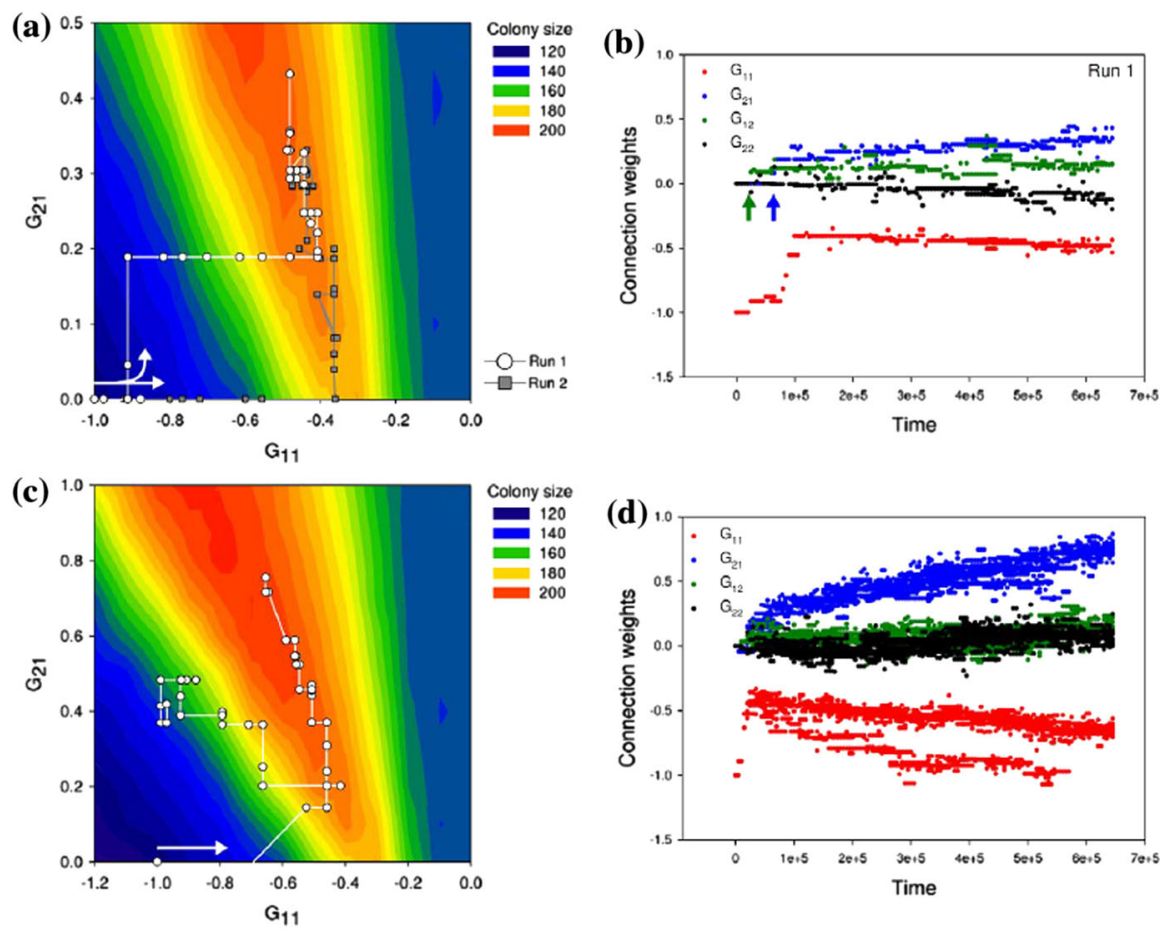

Fig. 13 Evolution of cell-to-cell communication under clonal and non-clonal growth conditions. The upper panel $\mathbf{a}$ and $\mathbf{b}$ shows the clonal growth conditions. The lower panel $\mathbf{c}$ and $\mathbf{d}$ shows the non-clonal growth conditions. The left plot in each panel a and c shows the evolutionary trajectory (evolution of cell-to-cell communication). Figure from van Gestel et al. (2012)

cules, and QS inhibitors. Individual cells are integrated into biomass particles and can produce EPS. Their setting contains several stochastic elements: size of the daughter particles at division, regulation, etc. To model QS, they assumed that the switch from down-regulated to up-regulated states is random but dependent of the concentration of the signalling substance in the containing voxel. QQ affects both the time of activation and QSM levels depending on when QSI was introduced: if introduced before activation, the proportion of up-regulated cells stays low for several hours before increasing; if introduced after activation, there is a higher proportion of up-regulated cells. This seems to be related to bistability in the system. They further explored the EPS production rate and how this affects QQ. Increasing EPS production leads to a thicker biofilm, making it more difficult for QSI to penetrate.

Cornforth et al. (2014) argued that bacteria use QS to infer both their social and physical properties of their environment simultaneously by combinatorially responding to multiple signalling molecules. They used a combination of approaches including a simple mathematical model for the extracellular dynamics of signal molecules in a well-mixed environment leading to a simple threshold behaviour. They also used an individual-based model to show that (1) differential decay and (2) combinatorial responses to two signals can provide better resolution when it comes to 
information regarding density and mass transfer. Using model selection, they identified several possible logic gates. From their study, they conclude that although QS (also commonly used to control secreted factors) seems more beneficial at high densities, under high-mass transfer conditions the digestive product will also be subjected to higher removal. Therefore, synergistic information on both cell density and mass transfer (and AND gate) is more beneficial. The AND gate limits the expression of costly secreted factors to the most beneficial high-density, low mass transfer environments.

Other models in this category worth to be mentioned are that from Goryachev et al. (2005), already reviewed in Goryachev (2011).

\subsubsection{Circuit and Feedback Loops Models}

A hybrid mathematical model combining stochastic simulation with frequency domain analysis showed that noise in QS signalling has the role to enable the entire bacterial population to engage in the positive feedback function of autoinduction. This is the model of Cox et al. (2003) for the QS system of V. fischeri. Given that the autoinducer concentration can make some individual cells to reach the apparent signal threshold earlier, this can lead to the production of additional autoinducer, therefore contributing to engaging those cells that had not reach the apparent threshold.

You et al. (2004) coupled gene expression to cell growth dynamics to investigate QS in a population of Escherichia coli. They designed a circuit which incorporates programmed death in response to changes in the environment (cell density). They used a simple mathematical model to show that their circuit can have a stable steady state in terms of cell density and gene expression that one can fine-tune by varying the stability of the cell-to-cell communication signal (expression of the LacZaCcdB killer protein). It could also have damped oscillations while approaching the steady state. Experiments confirmed the predictions of the model, although the circuit was found to be sensitive to perturbations. By varying the level of AHL degradation, it was possible to manipulate the system. Their model also predicted that intracellular levels of the killer protein would not be affected by changes in $\mathrm{pH}$. They discussed how indeed cell heterogeneity (in terms of size, age, plasmid copy number, gene expression, response to the killer protein) is actually required for the population control circuit to work.

Kuznetsov et al. (2004) constructed a genetic oscillator. The model consists of two subsystems: a toggle switch (which can displays bistability and hysteresis) and QS. It is a system of ODEs involving the cellular protein concentrations and active promoters as well as the autoinducer concentration. The first gives the condition for oscillations in isolated cells. There are basically two timescales, and they identified regions where a fast subsystem can be driven through a bistable region. They investigated these oscillations in more detail including how the requirements to obtain oscillations change if the cells interact. They claimed that strong interactions may result in suppression of synchronous oscillations. On the other hand, weak interactions may lead to oscillation but not synchronized. A large variation in the parameter values of the individual elements decreases the possibility of synchronized oscillations. 
An interesting theoretical paper showed that in a synthetic system of represilators QS, can be successfully used to create macroscopic rhythms (García-Ojalvo et al. 2004).

Perez et al. (2011) studied the noise and crosstalk between two acyl homoserine lactone signals (3OC6 HSL and C8HSL) in the QS mechanism of V.fisheri. Their mathematical model includes competitive-binding interactions between these two signals and describes the average (bulk culture) response of the lux operon to combinations of C8 HSL and 30C6 HSL signals. The first equation of their model was built on the basis of the power law and was able to predict different noise levels for luminescence versus fluorescence reporters of lux. They showed through several simulations that although the robust lux response to 30C6 HSL activation is highly heterogeneous (noisy), the presence of C8 HSL quenches the response largely by switching off the brightest cells. That is, the crosstalk does not seem to alleviate noise but tune sensitivity of the switch. They speculated that the QS circuit does not distinguish between different combinations of HSL inputs that produce the same average lux output.

Fujimoto and Sawai (2013) investigated whether QS could be graded (cells in the on and off states coexist within a population) or an all-or-none activation mechanism. The argue that QS circuits appear to be tuned so that the cells can use either type of transition depending on the environmental conditions. They used generic ODE models to describe QS across two levels of organization: single cell and cellular ensemble. They showed that graded transitions occur when the intracellular positive feedback alone can support bistability. All-or-none transitions occur when the secreted signal within the population serves predominantly to realize bistability at the group level.

There are many more models in this category, mainly computational models, we mentioned Tanouchi et al. (2008), Zhou et al. (2008), reviewed in Goryachev (2011). Another model on the topic of extracellular noise we would like to mention, but do not review due to the lack of space, is that of Hong et al. (2007).

\subsubsection{Heterogeneity in $Q S$}

There is increasing evidence that heterogeneity of QS activation occurs. This variability has been reported for bacterial species such as Vibrio fischeri (Anetzberger et al. 2009), Pseudomonas putida (Meyer et al. 2012), and Escherichia coli (Megerle et al. 2008). Furthermore, studies have focused on reporting that this heterogeneous distribution of gene expression is mainly observed when bacteria are grown in their natural habitat (Kang et al. 1999; Joyner and Lindow 2000) as opposed to well-mixed batch cultures, where QS has traditionally been analysed.

Meyer et al. (2012) used a microfluidic set-up to investigate Pseudomonas putida IsoF QS under flow and non-flow conditions at the single-cell level. Their experiments followed gene expression over time (using an AHL sensor). The mathematical model is an extension of Fekete et al. (2010) to a compartment model with interior and exterior space. They proposed two modelling approaches: a cell wall model (each cell constitutes a separate, but identical compartment, communicating via the external space) and compartment-barrier model (many bacteria are enclosed in a single large 


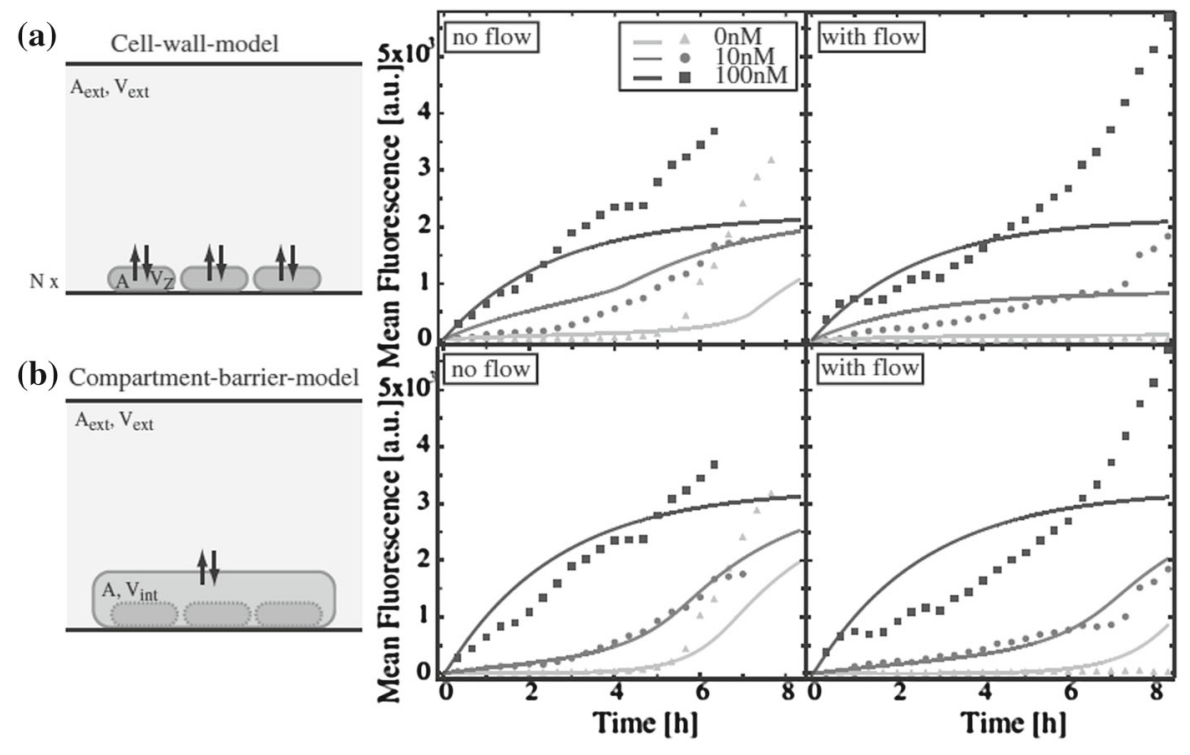

Fig. 14 Cell wall model a assumes that the cell membrane acts as an exchange barrier, while in the compartment-barrier model b, AHL can accumulate in a volume surrounding the bacteria. Expression kinetics at different external AHL concentrations $(0,10$, and $100 \mathrm{nM}$, without flow and with flow) and best fits of two different versions of the two-compartment model. The markers represent the average of the mean fluorescence of individual colonies, and the lines the corresponding model predictions. Figure from Meyer et al. (2012). Reproduced with permission

compartment, which is separated from the exterior). Their basic model is a system of ODEs for the number of bacteria, and for the time evolution of the fluorescence, the two distinct approaches are modelled in the third and fourth equations, for the internal and external AHL concentrations. They found that the non-fluorescent off state was reached in a slow exponential decay manner and showed that gene expression was suppressed by flow. The dynamics when AHL was added externally were similar under both conditions (with and without flow). Given that both modelling approaches reproduce the experimental observations reasonably well, they proposed that a hybrid model including transport mechanisms might improve the fit to the data. See Fig. 14.

Generally, Meyer study added to evidence of spatially heterogeneous gene expression and that QS regulation is not solely governed by cell density. They reported than variability was found to be more pronounced when AHLs were externally added to the media. Overall, their results suggested an extrinsic (external factors) rather than intrinsic (within the regulatory network) origin for this heterogeneity between colonies. They suggested division of work as a possible advantage/reason for this heterogeneity. This work is important because it provides further proof that QS systems give rise to complex, heterogeneous behaviour, rather than the common view of synchronous population response.

Hense et al. (2012) studied the issue of how QS generates spatially heterogeneous behaviour. They used a 3D QS model for attached microcolonies based on a luxtype system. They specifically looked at the influence of nutrients as an example of 
changing environmental factors. Their deterministic model consists of equation for the nutrients, AHL and cell concentration. They investigated the model behaviour with and without the influence of nutrients on the AHL production. Their aim was to promote a better understanding of the role of nutrients in QS activation. They solved the model numerically (PDEs) and discussed the ecological implications, including a hypothesis (pull-push approach) on why heterogeneities may be observed: local autoinducer concentration integrates both information on cells demand (pull) and cooperative potential (push). This is dependent on cell density, cell distribution, and mass transfer limitations. They suggested that the subpopulations generated by this heterogeneity (only possible in spatially structured populations) can be thought of division of work.

Pseudomonas syringae is a Gram-negative bacterium which lives on leaf surfaces. Pérez-Velázquez et al. (2015) used the integral of a non-negative stochastic process to study the QS state of bacterial colonies of P. syringae. They investigated the extent to which factors such as water availability and diffusional losses of QS signalling molecules would affect QS across colonies. Their results support that QS activation is indeed a good indicator of diffusional limitation, as QS is enhanced when diffusion of autoinducers signal decreases (either as a result of water availability or loss by diffusion). Using further experimental data, they explored heterogeneity of QS activation of this bacterium (colonies do not become homogeneously activated) when growing in this natural habitat. They were able to conclude that stochastic growth and uneven nutrient availability of the leaf surface may contribute only partially to the heterogeneity observed, discussing possible (evolutionary) explanations of this strategy.

\subsubsection{Cell-to-Cell Communication in Non-Bacterial Cells}

Almeida et al. (2012) proposed that $\mathrm{CD}^{+}{ }^{+} \mathrm{T}$ cells may be capable of cell-to-cell communication. It is known that these cells control their population numbers, but the mechanisms are not clear, more importantly how a constant balance (homoeostasis) is achieved, i.e. how do they "count" their number and "know" when to stop growing. The authors proposed that lymphocytes may be able (much the same as bacteria) to assess the number of molecules with which they interact and respond accordingly. In particular, they suggested that IL-2 (a cytokine signalling molecule in the immune system) producing cells may be involved in this process. QS in this case is defined as an indirect feedback loop, where $\mathrm{CD}^{+} \mathrm{T}$ cells sense the produced quantities of IL-2. This would explain how these cells regulate their number, in particular by restraining their growth. Moreover, they further hypothesize that a malfunction of this mechanism may lead to uncontrolled autoimmunity. A deterministic mathematical model to explore their hypotheses was presented. Their model describes the time evolution for four different CD4+T cells, and although the analysis is left for a further publication, they do however present some qualitative simulations (both for the deterministic model and for an stochastic version of it) to show the behaviour of their system, using arbitrary parameter values. In particular, how homoeostasis may be achieved.

Cancer stem cells (CSCs) differentiate or proliferate according to the feedback they receive from neighbouring cells. Vainstein et al. (2012) presented a simple discrete 
mathematical model for homoeostasis in developing tissues that is governed by a QS-like control mechanism, where stem cells differentiate or proliferate according to the feedback from the neighbouring cell populations. Previous analysis indicated that excessive stem cell proliferation leading to malignant transformation mainly results from altered sensitivity to micro-environmental signals. They analysed the dynamics of cancer cells and examined possible therapeutic approaches, e.g. by eliminating cancer stem cells (CSCs). They classified populations of cells in two categories, CSCs which can divide indefinitely and differentiated cancer cells, which do not divide and have a limited lifespan. They showed that neither stimulation of CSC differentiation nor inhibition of CSC proliferation alone is sufficient to completely eliminate CSCs. Only a combination of both can reduce population sizes. Their mathematical model consists of differentiation therapy and standard (cytotoxic or anti-proliferative) therapy. They investigated how these affect tumour progression separately and together, where they defined the cell behaviour stochastically.

\subsubsection{Others}

Müller and Uecker (2013) extended the work of Müller et al. (2006). They examined $N$ cells that communicate via a diffusive signalling substance. Linear diffusion equations were used to describe the dynamics outside the cell and an ODE was used to describe the dynamics inside the cell. The extracellular space and the intercellular space are linked via appropriate boundary conditions. The cells are represented as balls of radius $R$ in $\mathbb{R}^{3}$, and the aim was to investigate the limit $R \rightarrow 0$. They start by considering the case of a single cell and showed that the dynamics of the signalling substance (in the original model a integro-differential equation) can be approximated by solutions of an ODE. For $N \geq 2$, although it is possible to find an approximate ODE model, this cannot be easily scaled. They introduced a delta source for each cell which leads to a delayed (due to diffusion between different cells) system. They further discussed which approximation should be used in different cases (e.g. with more than one cell with $O(1)$ distances, the DDE model is a good approximation). They investigate QS by examining a colony of 27 cells arranged in a cubic pattern and explore how the distance between cells affects activation (if the distance between cells increases, we may or may not observed activation, including bistable regimes). The position of the cells is also relevant to the activation process, whereas the mass of signalling substances in the central cell may act as a trigger to activate other cells.

Brown (2013) challenged standard modelling approaches and proposed a new simple way to model QS systems, for both well-mixed and biofilm populations. Given that QS is a multiscale process, he claimed that the key is accurately take into consideration the interaction between molecular and population processes. His main assumption was that all QS systems share the following two processes: (1) there is a synthesis of the signal, and (2) there is transport of diffusion of this signal in and out of the cell. He assumed a well-mixed population of cells and then proposed one model for Gramnegative QS system (with intra- and extracellular signalling molecule concentration) and one for the Gram-positive one. His models relies on keeping track of the signal accumulation in compartments with different volumes. He added that models which do not do this may violate conservation of matter. He incorporated autoinduction using 
a Hill function. The derivation of the two model differs as follows: in Gram-positive bacteria, the intracellular signal refers to precursor peptide and extracellular signal to the AIP; transport occurs only in one direction, and the synthesis of the precursor peptide depends on the AIP concentration. He extended his model to incorporate a spatial model by considering a one-dimensional cross section of biofilm and approximate the population as a continuous population density. He further discussed the issue of bistability in QS systems both at the individual and population level, arguing that indeed heterogeneity (having a population where $80 \%$ of the cells are on and $20 \%$ are off) of activation is, unlike other models, predicted by his model. Furthermore, his spatial model can give rise to localized QS regions within the biofilm.

Uecker et al. (2014) presented a model of QS when background flow is present. It describes cells with fixed locations in a laminar flow with constant velocity. It follows Müller et al. (2006) and Müller and Uecker (2013). It examined the time evolution of the internal state of the cell (through ODEs) and an external one (tube subjected to background laminar flow) where the signalling substance diffuses. In this context, the signalling substance can be either washed out or accumulated downstream. They found that washout causes communication to break down rather than causing activation due to downstream effect.

Gölgeli Matur et al. (2014) addressed the QS mechanism of single cells in the stationary case, which is crucial to understanding the effect of chemical gradients and inter-cell variations. Based on a reaction-diffusion system, they developed a method that drastically reduces the computational complexity of the model. They obtained approximative algebraic equations through a suitable scaling. They simulated a scenario for different spatial cell arrangements that explores the question of microscale communication. Their approach allows computing the signalling substance distribution in real-world problems, such as for the pathogenic bacteria Pseudomonas aeruginosa in (spatially unlimited) lung mucus (see Fig. 15).

Other mathematical models reviewed in Goryachev (2011) are Bischofs et al. (2009). He also has a section on QS in synthetic systems, including McMillen et al. (2002), Koseska et al. (2007), Ullner et al. (2007), Danino et al. (2010), Chen and Weiss (2005), which we do not cover here.

Overview and Future Research Directions Given that QS controls a vast range of target functions and, in nature, takes place in complex environments, mathematical models of QS require the use of novel and diverse techniques. In particular, models should be able to integrate the role of the conditions in which QS takes place, i.e. the ecological context and life history of the bacteria now known to play a pivotal role in QS systems. Models that take into consideration both spatial configuration (such as agent-based models) and evolutionary dynamics (for instance adaptive dynamics) are in principle well equipped to address this complexity. Agent-based models have the additional advantage of enabling the investigation of QS at the single-cell level. Some of the models of Sect. 2.5 addressed these issues (Sect. 2.5.1).

Given that autoinduction often depends on the metabolic state of the cell, models that can describe a QS circuit but are unable to determine how this circuit depends on other processes fail to completely understand an AI system. Models including the interaction of the QS regulation system with its ecological function, specifically 


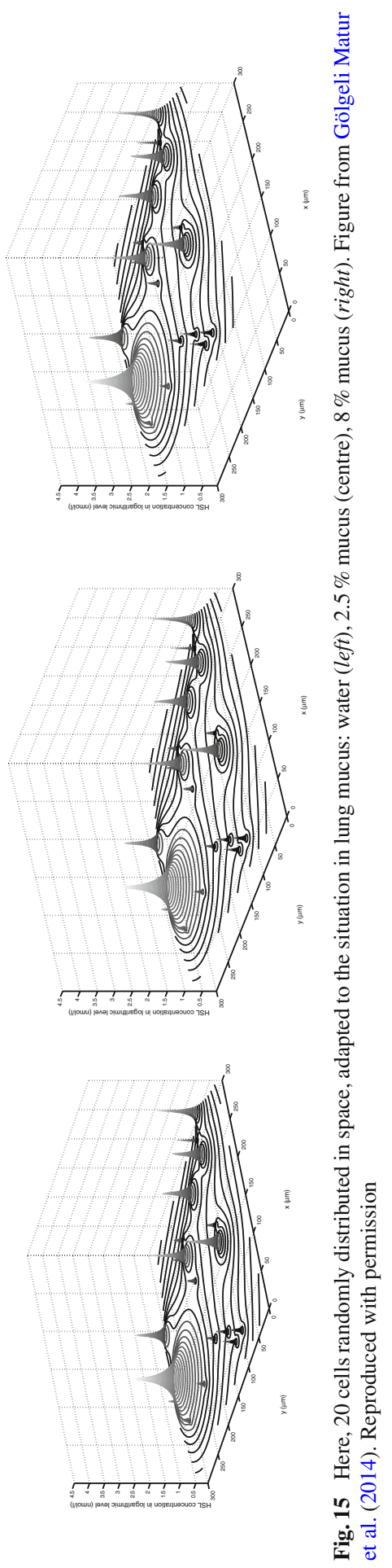


in systems with several interconnected AI systems, should be further developed. $B$. subtilis is an example of this complexity as its signalling network differentiates cells into at least seven subtypes.

Recent findings have led to the re-evaluation of the classical QS paradigm (synchronous all-or-none behaviour of the population): a graded response at the population level is also possible. QS does not necessarily work under synchronous, uniform responses of all cells in a population. Chemical gradients or stochastic variations may lead to heterogeneous QS activation. In such cases, QS activation is nonsynchronous and heterogeneous yet cooperative and coordinated such that it benefits the whole population. Models addressing this, similar to the work of Fujimoto and Sawai (2013), provide interesting future research directions on QS broad range of operation.

Physical properties of the environment in which QS takes place are pivotal, for example how they affect solubility and degradation of AIs. Additionally, these conditions may vary in time and space. Müller or Brown's approaches are important distinct steps in this direction. Mechanical and multiphase models may also be well equipped to describe the role of these properties.

The van Gastel et al.'s model successfully includes both evolutionary and environmental aspects of QS, which makes it a well-rounded model. It adds to the understanding of QS as a cost and benefit predictor. Under certain circumstances, it may be crucial for bacteria to anticipate environmental conditions, whereas in other circumstances it may be ecologically irrelevant (e.g. cells living under constant, predictable mass transfer conditions). Their model is relevant in cases where QS activation constitutes a lifetime switch, from resident to pathogenic, for example. In this case, QS evolutionary benefits are strongly coupled with efficient assessment of environmental changes.

Heterogeneity in QS is now very well documented. Typically, in QS active populations, there is a fraction of non-contributors. In spatially structured populations (e.g. biofilms), physiochemical gradients cause this heterogeneity, but the precise mechanisms and functions are not well understood. Mathematical models, including molecular noise and stochasticity, are a step forward to describe heterogeneity of QS.

In terms of future research directions, we would like to highlight two potential areas which in our view deserve more attention in terms of novel approaches:

- the fact that environmental factors are known to directly affect QS. Temperature and pH influence the stability of QS molecules; other bacterial or eukaryotic species may degrade or block AIs. These factors naturally affect what QS “measures" and for what purpose. Mathematical models which enable the incorporation of these consideration may prove really useful.

- division of labour/multiple AI systems. In B. subtilis for example, both competence and sporulation are QS-regulated, but they cannot work simultaneously in the same cell; therefore a "division of labour" may take place. This mounting evidence of complexity requires ad hoc tools such as mathematical modelling, for example for the underlying circuits of the multiple AI systems as they may involve different AI thresholds for different phenotypes. 


\section{Future Challenges and Discussion}

We conclude this review by discussing the future potential of modelling in QS as well as current research challenges.

Remarkable progress has been made towards an integrative (theoretical and experimental) understanding of bacterial communication. The mathematical modelling of QS involves a variety of modelling approaches, depending on the subject of exploration. These models may include AIs production and transport, cellular growth and division dynamics. Models can be deterministic (when describing the AI dynamics, for example) or stochastic (when describing heterogeneity of activation). Two approaches have been used to describe QS activation. The first divides the population into up-regulated and down-regulated subpopulations (Ward et al. 2001), whereas the second option is to focus on the concentration of AIs (Dockery and Keener 2001). Often, a time delay occurs in bacterial gene regulation (Barbarossa et al. (2010). In deterministic approaches, the activity and production of signalling molecules (Dockery and Keener 2001), the way they bind to the promoter gene region (Goryachev et al. 2006), and the feedback loops (Frederick et al. 2011) are often described by an ODE system at the population level. A rough classification of all presented models by way of summary can be found in Table 3 .

Bacterial communication could mean several things, from autoinduction (cells sense and perceive their own signal) to communication from a more classical viewpoint (one cell type is a signal sender while another is a receiver). In this review, we concentrated on QS (autoinduction) and refer the reader to Hense and Schuster (2015) for a more generalist discussion.

What is known as QS has turned out to be a highly sophisticated mechanism employed by bacteria to regulate gene expression. It is now widely accepted that bacterial communication provides information on population (social) as well as physical properties of the environment, i.e. both density and diffusion matter. Several studies have now shown that apart from its recognized group benefits, QS can also operate at the single-cell level (Boedicker et al. 2009; Carnes et al. 2010; Whitaker et al. 2011). Generally, we can name two properties common to most QS systems: (1) auto-regulation and (2) cooperativity. The interdependencies of these properties in particular in relation to its ecological function is central to understanding QS.

The nature of this autocrine type of communication has allowed researchers to use mathematical as well as computational approaches to analyse specific and global properties of several QS systems. In many cases, with the help of technological development, it has been possible to test experimentally these models. The field of mathematical modelling of QS has been fortunate enough to benefit from close cooperation with microbiologists to further our understanding of how bacteria communicate. Most of the works reviewed here have been interdisciplinary efforts, culminating in successful integrative approaches. In several cases, the use of mathematical models was crucial.

Several important questions still remain. The ecological context of QS is key to understanding its function. What appears as social cheating or non-cooperative behaviour at first may actually be viewed as altruistic when interpreted in the appropriate ecological context (for instance, detachment of cells from biofilms). Moreover, 


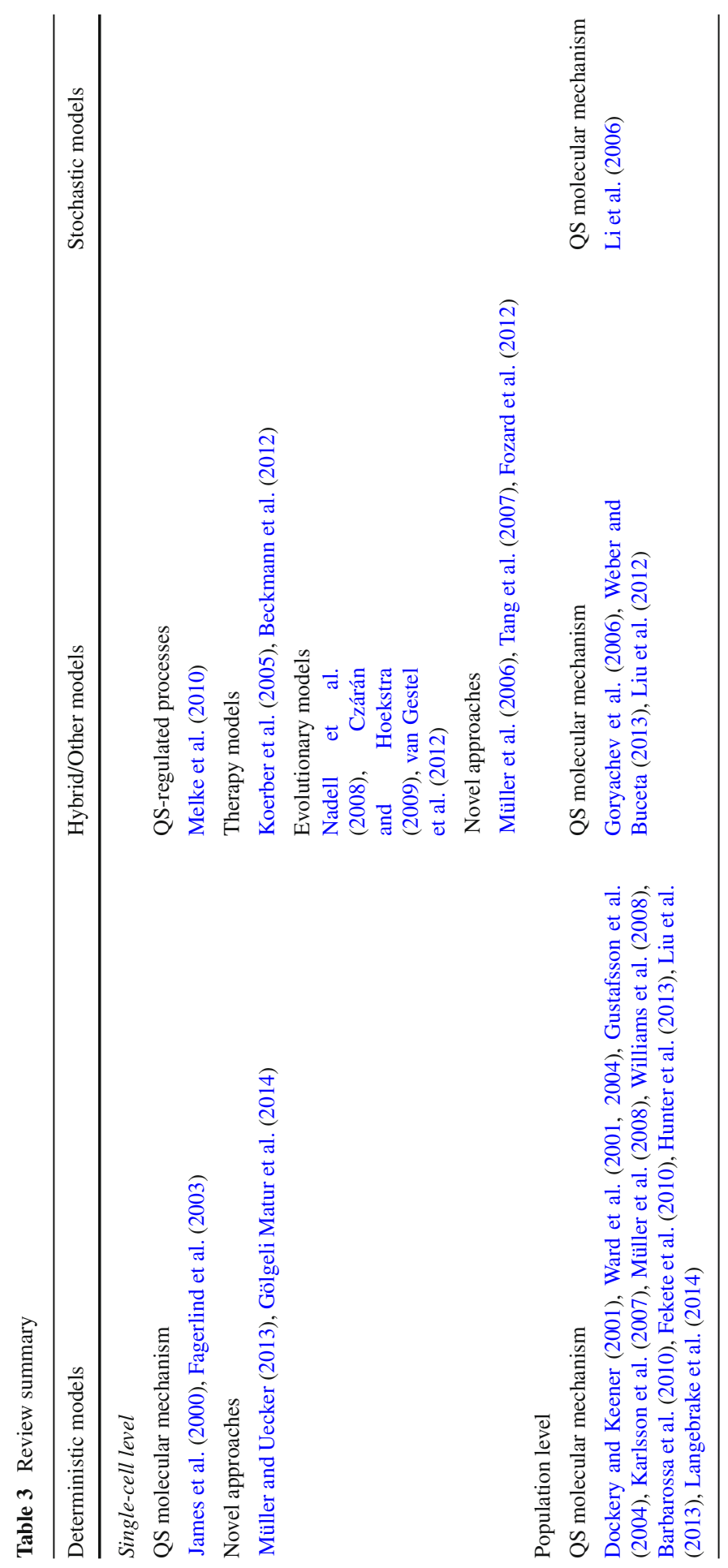




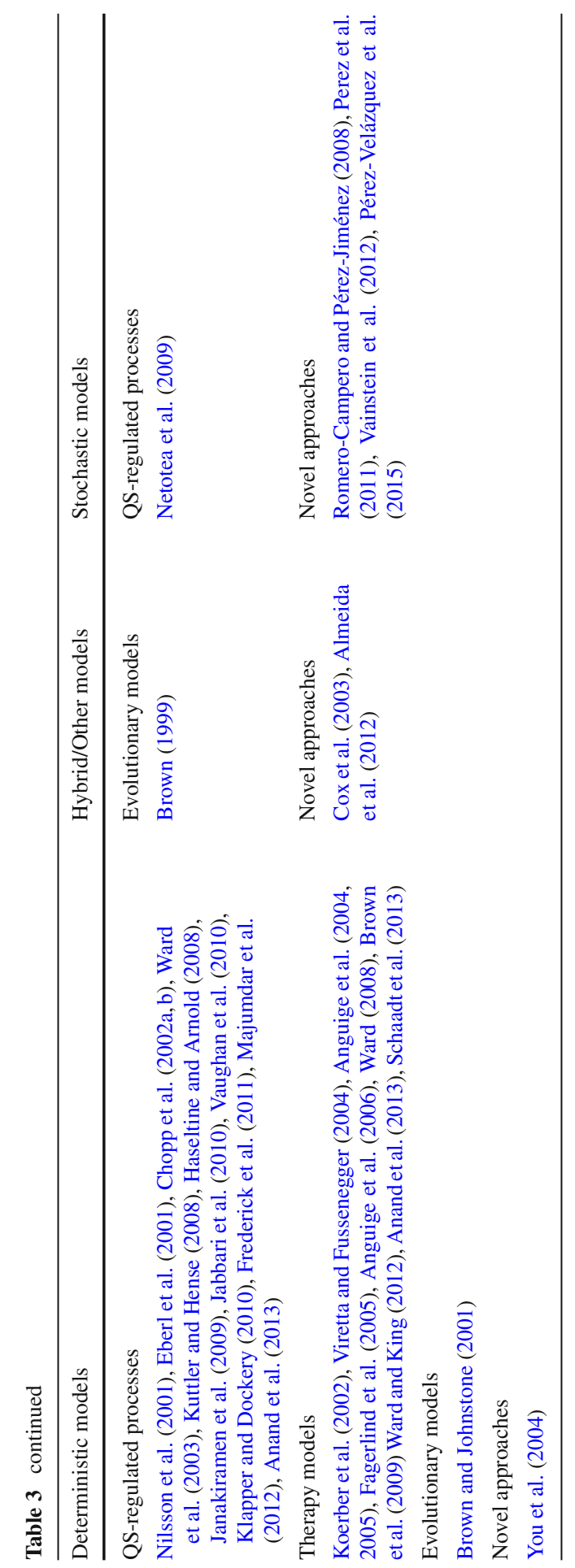


there exist examples of QS triggering non-cooperative behaviour. Hense and Schuster (2015) argue that QS regulates cooperative behaviour, but specific circumstances may not always allow for cooperation, i.e. it is the situation that determines whether a behaviour is cooperative and not the behaviour itself.

The development of new experimental and image analysing techniques is making it possible to elucidate more intricate features of cell-to-cell bacterial communication, such as combinatorial QS (Cornforth et al. 2014), phenotypic switch (Dumas et al. 2013), and reversible non-genetic phenotypic heterogeneity (Pradhan and Chatterjee 2014). As a sub-area of mathematical biology, mathematical modelling of QS represents an exciting and vast research area with rich potential for interdisciplinary collaboration.

The classical interpretation of QS as a cell-density-controlled mechanism has now been broadened. The relevance of spatial distribution and mass transfer properties has now been confirmed experimentally. QS is nowadays understood to have a variety of ecological functions that differ according to the species and the environment where it takes place.

Extracellular factors could affect QS systems, e.g. in V. fischeri, nutrient deficiency ceases QS. This research avenue has not been explored broadly with mathematical models and offers potentially rich possibilities: for example, in extreme stressful situations, activation may lead bacteria to commit suicide for the benefit of others.

Spatial, hybrid, and multiscale models represent useful techniques to address QS. Spatial location may represent specific demands at the single cell level but should translate in integrated responses at the population level. Agent-based models also permit the inclusion of environmental factors (such as $\mathrm{pH}$ and temperature), the presence of competing or beneficial microbes, and the developmental state of the cell (such as sporulation and mobility).

The ability of a mathematical model to predict depends heavily on its parametrization, and therefore, obtaining suitable data from in vivo and in vitro QS systems is essential. With new experimental data available, some assumptions made in mathematical models have been corroborated or disclaimed. For example, it has been shown that low nutrient availability does decrease growth but increases QS gene expression per cell. QS systems require information about the physical properties of the environment to enable both isolated cells and groups to optimize their behaviour accordingly. Spatio-temporal data of QSM concentrations are highly desirable for the validation of mathematical models. The role of QQ enzymes should also be explored further with modelling. It has been suggested they may regulate inter-colony communication. Current models of QQ are relatively descriptive, mostly owing to the lack of suitable data.

Increasing complexity in the model does not necessarily lead to a better understanding of QS. The auto-regulatory nature of QS often leads to including a positive feedback loop in the associated mathematical model. More complex architectures predict bistability, which has been assumed to depend on the stability of receptor-AI complexes but has only rarely been confirmed experimentally.

It remains unclear how best to investigate the evolutionary purpose of QS. It has been proposed that, originally, AIs may not have been released to detect cell density but simply, e.g. as waste products, and that eventually bacterial cells realized the 
advantages of being endowed with information of the local concentration of these substances, leading to the evolution of QS as we know it today. Models addressing this evolutionary purpose represent an interesting research avenue.

Quantitative approaches that help exploring novel therapies and treatments, such as phage therapy in combination with either antibacterial or QQ therapies, are needed. This also applies to the investigation of the interaction of QS with the host.

The use of mathematics to understanding QS has given rise to new analytical tools and novel modelling approaches. It has also helped addressing certain gaps to comprehensively investigate QS, from the ecological context to gene regulation. We believe mathematical modelling of QS has been instrumental in this field and will continue to play a key role.

Acknowledgments RGC was supported by a grant from SEP/CONACyT-Mexico No. 152794. JPV wants to thank Stephen Starck (TUM) for his patience and help revising the document.

\section{References}

Almeida AR, Amado IF, Reynolds J, Berges J, Lythe G, Molina-Paris C, Freitas AA (2012) Quorum sensing in CD4+ T cell homoeostasis: a hypothesis and a model. Front. Immunol. 3. doi:10.3389/fimmu.2012. 00125

Anand R, Rai N, Thattai M (2013) Interactions among quorum sensing inhibitors. PLOS One 8:e62254

Anetzberger C, Pirch T, Jung K (2009) Heterogeneity in quorum sensing-regulated bioluminescence of Vibrio harveyi. Mol Microbiol 73:267-277. doi:10.1111/j.1365-2958.2009.06768.x

Anguige K, King JR, Ward JP (2005) Modelling antibiotic- and anti-quorum sensing treatment of a spatially-structured Pseudomonas aeruginosa population. J Math Biol 51(5):557-594. doi:10.1007/ s00285-005-0316-8

Anguige K, King JR, Ward JP (2006) A multi-phase mathematical model of quorum sensing in a maturing Pseudomonas aeruginosa biofilm. Math Biosci 203:240-276. doi:10.1016/j.mbs.2006.05.009

Anguige K, King JR, Ward JP, Williams P (2004) Mathematical modelling of therapies targeted at bacterial quorum sensing. Math Biosci 192(1):39-83. doi:10.1016/j.mbs.2004.06.008

Banik SK, Fenley AT, Kulkarni RV (2009) A model for signal transduction during quorum sensing in Vibrio harveyi. Phys Biol 6. doi:10.1088/1478-3975/6/4/046008

Barbarossa M, Kuttler C, Fekete A, Rothballer M (2010) A delay model for quorum sensing of Pseudomonas putida. Biosystems 102:148-156. doi:10.1016/j.biosystems.2010.09.001

Beckmann BE, Knoester DB, Connelly BD, Waters CM, McKinley PK (2012) Evolution of resistance to quorum quenching in digital organisms. Artif Life 18:291-310. doi:10.1162/artl_a_00066

Ben-Jacob E, Cohen I, Levine H (2000) Cooperative self-organization of microorganisms. Adv Phys 49:395554. doi:10.1080/000187300405228

Bischofs IB, Hug JA, Liu AW, Wolf DM, Arkin AP (2009) Complexity in bacterial cell-cell communication: quorum signal integration and subpopulation signaling in the Bacillus subtilis phosphorelay. Proc Natl Acad Sci USA 106:6459-6464. doi:10.1073/pnas.0810878106

Boyer M, Wisniewski-Dyé F (2009) Cell-cell signalling in bacteria: not simply a matter of quorum. FEMS Microbiol Ecol 70:1-9. doi:10.1111/j.1574-6941.2009.00745.x

Boedicker J, Vincent M, Ismagilov R (2009) Microfluidic confinement of single cells of bacteria in small volumes initiates high-density behavior of quorum sensing and growth and reveals its variability. Angew Chem Int Ed 48:5908-5911. doi:10.1002/anie.200901550

Brookfield J (1998) Quorum sensing and group selection. Evolution 52:1263-1269

Brown D (2010) A mathematical model of the gac/rsm quorum sensing network in Pseudomonas fluorescens. Biosystems 101:200-212. doi:10.1016/j.biosystems.2010.07.004

Brown D (2013) Linking molecular and population processes in mathematical models of quorum sensing. Bull Math Biol 75:1813-1839. doi:10.1007/s11538-013-9870-1

Brown SP (1999) Cooperation and conflict in host-manipulating parasites. Proc R Soc B 266:1899-1899. doi:10.1098/rspb.1999.0864 
Brown SP, Johnstone RA (2001) Cooperation in the dark: signalling and collective action in quorum-sensing bacteria. Proc R Soc B 268:961-965. doi:10.1098/rspb.2001.1609

Brown SP, West SA, Diggle SP, Griffin AS (2009) Social evolution in micro-organisms and a trojan horse approach to medical intervention strategies. Philos Trans R Soc B 364:3157-3168. doi:10.1098/rstb. 2009.0055

Busby S, de Lorenzo V (2001) Cell regulation: putting together pieces of the big puzzle. Curr Opin Microbiol 4:117-118. doi:10.1016/S1369-5274(00)00175-2

Calfee MW, Coleman JP, Pesci EC (2001) Interference with Pseudomonas quinolone signal synthesis inhibits virulence factor expression by Pseudomonas aeruginosa. Proc Natl Acad Sci USA 98:11633-11637

Carnes EC, Lopez DM, Donegan NP, Cheung A, Gresham H, Timmins GS, Brinker (2010) Confinementinduced quorum sensing of individual Staphylococcus aureus bacteria. Nat Chem Biol 6, 41-45. URL: http://www.ncbi.nlm.nih.gov/pubmed/19935660

Castillo-Juarez I, Maeda T, Mandujano-Tinoco EA, Tomas M, Perez-Eretza B, Garcia-Contreras R (2015) Role of quorum sensing in bacterial infections. World J Clin Cases 3:575-598

Chen CC, Riadi L, Suh SJ, Ohman DE, Ju LK (2005) Degradation and synthesis kinetics of quorumsensing autoinducer in Pseudomonas aeruginosa cultivation. J Biotechnol 117:1-10. doi:10.1016/j. jbiotec.2005.01.003

Chen F, Chen CC, Riadi L, Ju LK (2004) Role of quorum sensing in bacterial infections. Biotechnol Prog 20:1325-1331. doi:10.1021/bp049928b

Chen MT, Weiss R (2005) Artificial cell-cell communication in yeast Saccharomyces cerevisiae using signaling elements from Arabidopsis thaliana. Nat Biotechnol 23:1551-1555. doi:10.1038/nbt1162

Chopp DL, Kirisits MJ, Moran B, Parsek MR (2002a) The dependence of quorum sensing on the depth of a growing biofilm. Bull Math Biol 65:1053-1079

Chopp DL, Kirisits MJ, Moran B, Parsek MR (2002b) A mathematical model for quorum sensing in a growing bacterial biofilm. J Ind Microbiol Biotechnol 296:339-346

Cornforth DM, Popat R, McNally L, Gurney J, Scott-Phillips TC, Ivens A, Diggle SP, Brown SP (2014) Combinatorial quorum sensing allows bacteria to resolve their social and physical environment. Proc Natl Acad Sci USA 111:4280-4284. doi:10.1073/pnas.1319175111

Costerton JW, Stewart P, Greenberg EP (1999) Bacterial biofilms: a common cause of persistent infections. Science 284:1318-1322

Cox CD, Peterson GD, Allen MS, Lancaster JM, McCollum JM, Austin D, Yan L, Sayler GS, Simpson ML (2003) Analysis of noise in quorum sensing. OMICS 7:317-334. doi:10.1089/153623103322452422

Czárán T, Hoekstra RF (2009) Microbial communication, cooperation and cheating: Quorum sensing drives the evolution of cooperation in bacteria. PLoS One 4:e6655. doi:10.1371/journal.pone.0006655

Damore JA, Gore J (2012) Understanding microbial cooperation. J Theor Biol 299:31-41. doi:10.1016/j. jtbi.2011.03.008

Danino T, Mondragon-Palomino O, Tsimring L, Hasty J (2010) A synchronized quorum of genetic clocks. Nature 463:326-330. doi:10.1038/nature08753

Davies DSC (2011) Annual report of the chief medical officer. Technical Report. British Department of Health

Dilanji GE, Langebrake JB, De Leenheer P, Hagen SJ (2012) Quorum activation at a distance: spatiotemporal patterns of gene regulation from diffusion of an autoinducer signal. J Am Chem Soc 134:5618-5626. doi: $10.1021 / \mathrm{ja} 211593 \mathrm{q}$

Dockery J, Keener J (2001) A mathematical model for quorum sensing in Pseudomonas aeruginosa. Bull Math Biol 63:95-116. doi:10.1006/bulm.2000.0205

Dong YH, Gusti AR, Zhang Q, Xu JL, Zhang LH (2002) Identification of quorum-quenching N-acyl homoserine lactonases from Bacillus species. Appl Environ Microbiol 68:1754-1759. doi:10.1128/ AEM.68.4.1754-1759.2002

Duddu R, Chopp DL, Moran B (2009) A two-dimensional continuum model of biofilm growth incorporating fluid flow and shear stress based detachment. Biotechnol Bioeng 103:92-104. doi:10.1002/bit.22233

Dumas Z, Ross-Gillespie A, Kmmerli R (2013) Switching between apparently redundant iron-uptake mechanisms benefits bacteria in changeable environments. Proc R Soc B 280. doi:10.1098/rspb.2013.1055

Eberl H, Parker D, van Loosdrecht M (2001) A new deterministic spatio-temporal continuum model for biofilm development. J Theor Med 3:161-175. doi:10.1080/10273660108833072

Eberl H, Morgenroth E, Noguera D, Picioreanu C, Rittmann B, van Loosdrecht M, Wanner O (2006) Mathematical modeling of biofilms. Scientific and technical report, IWA Publishing. URL: https:// books.google.de/books?id=hRsRy6jSKKAC 
Fagerlind MG, Nilsson P, Harlén M, Karlsson S, Rice SA, Kjelleberg S (2005) Modeling the effect of acylated homoserine lactone antagonists in Pseudomonas aeruginosa. Biosystems 80:201-213. doi:10.1016/j. biosystems.2004.11.008

Fagerlind MG, Rice SA, Nilsson P, Harlén M, James S, Charlton T, Kjelleberg S (2003) The role of regulators in the expression of quorum-sensing signals in Pseudomonas aeruginosa. J Mol Microbiol Biotechnol 6:88-100. doi: 10.1159/000076739

Fekete A, Kuttler C, Rothballer M, Hense BA, Fischer D, Buddrus-Schiemann K, Lucio M, Müller J, Schmitt-Kopplin P, Hartmann A (2010) Dynamic regulation of n-acyl-homoserine lactone production and degradation in Pseudomonas putida ISOF. FEMS Microbiol Ecol 72:22-34. doi:10.1111/j. 1574-6941.2009.00828.x

Fenley AT, Banik SK, Kulkarni RV (2011) Computational modeling of differences in the quorum sensing induced luminescence phenotypes of Vibrio harveyi and Vibrio cholerae. J Theor Biol 274(1):145-153. doi:10.1016/j.jtbi.2011.01.008

Fletcher JA, Doebeli M (2009) A simple and general explanation for the evolution of altruism. Proc R Soc B 276:13-19

Fozard J, Lees M, King J, Logan B (2012) Inhibition of quorum sensing in a computational biofilm simulation. Biosystems 109:105-114. doi:10.1016/j.biosystems.2012.02.002

Frederick M, Kuttler C, Hense B, Müller J, Eberl H (2010) A mathematical model of quorum sensing in patchy biofilm communities with slow background flow. Can Appl Math Q 18:267-298

Frederick MR, Kuttler C, Hense BA, Eberl HJ (2011) A mathematical model of quorum sensing regulated eps production in biofilm communities. Theor Biol Med Model. 8. doi:10.1186/1742-4682-8-8

Friman VP, Diggle SP, Buckling A (2013) Protist predation can favour cooperation within bacterial species. Biol Lett 9. doi:10.1098/rsbl.2013.0548

Friman VP, Ghoul M, Molin S, Johansen HK, Buckling A (2013b) Pseudomonas aeruginosa adaptation to lungs of cystic fibrosis patients leads to lowered resistance to phage and protist enemies. PLoS One 8:e75380. doi:10.1371/journal.pone.0075380

Fujimoto K, Sawai S (2013) A design principle of group-level decision making in cell populations. PLoS Comput Biol 9:e1003110. doi:10.1371/journal.pcbi.1003110

Fuqua WC, Winans SC, Greenberg EP (1994) Quorum sensing in bacteria - the luxr-luxi family of cell density-responsive transcriptional regulators. J Bacteriol 176:269-275

Galloway WR, Hodgkinson JT, Bowden S, Welch M, Spring DR (2012) Applications of small molecule activators and inhibitors of quorum sensing in Gram-negative bacteria. Trends Microbiol 20:449-458. doi:10.1016/j.tim.2012.06.003

García-Contreras R, Maeda T, Wood TK (2013) Resistance to quorum-quenching compounds. Appl Environ Microbiol 79:6840-6846. doi:10.1128/AEM.02378-13

García-Contreras R, Maeda T, Wood TK (2015) Can resistance against quorum-sensing interference be selected?. ISME J 1751-7370. URL: http://www.nature.com/ismej/journal/vaop/ncurrent/full/ ismej201584a.html

García-Contreras R, Nunez-Lopez L, Jasso-Chavez R, Kwan BW, Belmont JA, Rangel-Vega A, Maeda T, Wood TK (2015) Quorum sensing enhancement of the stress response promotes resistance to quorum quenching and prevents social cheating. ISME J 9:115-125. doi:10.1038/ismej.2014.98

García-Ojalvo J, Elowitz MB, Strogatz SH (2004) Modeling a synthetic multicellular clock: repressilators coupled by quorum sensing. Proc Natl Acad Sci USA 101:10955-10960. doi:10.1073/pnas. 0307095101

Gillespie DT (1977) Exact stochastic simulation of coupled chemical reactions. J Phys Chem A 81:23402361. doi:10.1021/j100540a008

Gölgeli Matur M, Müller J, Kuttler C, Hense BA (2014) An approximative approach for single cell spatial modeling of quorum sensing. J Comput Biol. doi:10.1089/cmb.2014.0198

González-Barrios AF, Covo V, Medina LM, Vives-Florez M, Achenie L (2009) Quorum quenching analysis in Pseudomonas aeruginosa and Escherichia coli: network topology and inhibition mechanism effect on the optimized inhibitor dose. Bioprocess Biosyst Eng 32:545-556. doi:10.1007/s00449-008-0276-7

González-Barrios AF, Achenie LE (2010) Escherichia coli autoinducer-2 uptake network does not display hysteretic behavior but ai-2 synthesis rate controls transient bifurcation. Biosystems 99:17-26. doi:10. 1016/j.biosystems.2009.08.003

Goryachev AB (2009) Design principles of the bacterial quorum sensing gene networks. Wiley Interdiscip Rev Syst Biol Med 1:45-60. doi:10.1002/wsbm.27 
Goryachev AB (2011) Understanding bacterial cell-cell communication with computational modeling. Chem Rev 111:238-250. doi:10.1021/cr100286z

Goryachev AB, Toh DJ, Lee T (2006) Systems analysis of a quorum sensing network: Design constraints imposed by the functional requirements, network topology and kinetic constants. BioSystems 83: 178-187. URL: http://goryachev.bio.ed.ac.uk/goryachev/sites/sbsweb2.bio.ed.ac.uk.goryachev/files/ pdf/BioSys0206

Goryachev AB, Toh DJ, Wee KB, Lee T, Zhang HB, Zhang LH (2005) Transition to quorum sensing in an agrobacterium population: a stochastic model. PLoS Comput Biol 1(4):e37. doi:10.1371/journal. pcbi.0010037

Goss PJE, Peccoud J (1998) Quantitative modeling of stochastic systems in molecular biology by using stochastic petri nets. Proc Natl Acad Sci USA 95:6750-6755. URL: http://www.pnas.org/content/95/ 12/6750.abstract

Gupta P, Chhibber S, Harjai K (2015) Efficacy of purified lactonase and ciprofloxacin in preventing systemic spread of Pseudomonas aeruginosa in murine burn wound model. Burns 41:153-162

Gustafsson E, Nilsson P, Karlsson S, Arvidson S (2004) Characterizing the dynamics of the quorum-sensing system in Staphylococcus aureus. J Mol Microbiol Biotechnol 8:232-242. doi: 10.1159/000086704

Hamilton WD (1964) The genetical evolution of social behaviour. J Theor Biol 7:1-16

Haseltine EL, Arnold FH (2008) Implications of rewiring bacterial quorum sensing. Appl Environ Microbiol 74:437-445

Hastings JW, Nealson KH (1977) Bacterial bioluminescence. Annu Rev Microbiol 31:549-595. doi:10. 1146/annurev.mi.31.100177.003001

Hense BA, Kuttler C, Müller J, Rothballer M, Hartmann A, Kreft JU (2007) Does efficiency sensing unify diffusion and quorum sensing? Nat Rev Microbiol 5:230-239. doi:10.1038/nrmicro1600

Hense BA, Müller J, Kuttler C, Hartmann A (2012) Spatial heterogeneity of autoinducer regulation systems. Sensors 12(4):4156-4171. doi:10.3390/s120404156

Hense BA, Schuster M (2015) Core principles of bacterial autoinducer systems. Microbiol Mol Biol Rev 79:153-169. doi:10.1128/MMBR.00024-14

Hong D, Saidel WM, Man S, Martin JV (2007) Extracellular noise-induced stochastic synchronization in heterogeneous quorum sensing network. J Theor Biol 245:726-736. URL: http://www.sciencedirect. com/science/article/pii/S0022519306005662

Hunter GAM, Vasquez FG, Keener JP (2013) A mathematical model and quantitative comparison of the small RNA circuit in the Vibrio harveyi and Vibrio cholerae quorum sensing systems. Phys Biol 10:046007. URL: http://stacks.iop.org/1478-3975/10/i=4/a=046007

Jabbari S, King JR, Koerber AJ, Williams P (2010) Mathematical modelling of the agr operon in Staphylococcus aureus. J Math Biol 61:17-54

James S, Nilsson P, James G, Kjelleberg S, Fagersötrm T (2000) Luminescence control in the marine bacterium Vibrio fischeri: an analysis of the dynamics of lux regulation. J Mol Biol 296:1127-1137. doi:10.1006/jmbi.1999.3484

Janakiramen V, Englert D, Jayaman A, Baskaran H (2009) Modeling growth and quorum sensing in biofilms grown in microfluidic chambers. Ann Biomed Eng 37:1206-1216

Joyner DC, Lindow SE (2000) Heterogeneity of iron bioavailability on plants assessed with a wholecell GFP-based bacterial biosensor. Microbiology 146:2435-2445. URL: http://mic.sgmjournals.org/ content/146/10/2435.abstract

Kaplan HB, Greenberg EP (1985) Diffusion of autoinducer is involved in regulation of the Vibrio fischeri luminescence system. J Bacteriol 163:1210-1214. URL: http://jb.asm.org/content/163/3/1210. abstract

Kang Y, Saile E, Schell MA, Denny TP (1999) Quantitative immunofluorescence of regulated EPS gene expression in single cells of Ralstonia solanacearum. Appl Environ Microbiol 65:2356-2362. URL: http://aem.asm.org/content/65/6/2356.abstract

Karlsson D, Karlsson S, Gustafsson E, Normark BH, Nilsson P (2007) Modeling the regulation of the competence-evoking quorum sensing network in Streptococcus pneumoniae. BioSystems 90:211223

Kirisits MJ, Margolis JJ, Purevdorj-Gage BL, Vaughan B, Chopp DL, Stoodley P, Parsek MR (2007) Influence of the hydrodynamic environment on quorum sensing in Pseudomonas aeruginosa biofilms. J Bacteriol 189:8357-8360. doi:10.1128/JB.01040-07

Klapper I, Dockery J (2010) Mathematical description of microbial biofilms. SIAM Rev 52:000-000 
Koerber A, King J, Ward J, Williams P, Croft J, Sockett R (2002) A mathematical model of partial-thickness burn-wound infection by Pseudomonas aeruginosa: quorum sensing and the build-up to invasion. Bull Math Biol 64:239-259. doi:10.1006/bulm.2001.0272

Koerber AJ, King JR, Williams P (2005) Deterministic and stochastic modelling of endosome escape by Staphylococcus aureus: quorum sensing by a single bacterium. J Math Biol 50(4):440-488. doi:10. 1007/s00285-004-0296-0

Koseska A, Volkov E, Zaikin A, Kurths J (2007) Quantized cycling time in artificial gene networks induced by noise and intercell communication. Phys Rev E 76:020901. doi:10.1103/PhysRevE.76.020901

Kumar S, Kolodkin-Gal I, Engelberg-Kulka H (2013) Novel quorum sensing peptides mediating interspecies bacterial cell death. MBio 4(3):e00314-13. doi:10.1128/mBio.00314-13

Kuttler C, Hense BA (2008) Interplay of two quorum sensing regulation systems of Vibrio fischeri. J Theor Biol 251:167-180. doi:10.1016/j.jtbi.2007.11.015

Kuznetsov A, Krn M, Kopell N (2004) Synchrony in a population of hysteresis-based genetic oscillators. SIAM J Appl Math 65

Langebrake JB, Dilanji GE, Hagen SJ, Leenheer PD (2014) Traveling waves in response to a diffusing quorum sensing signal in spatially-extended bacterial colonies. J Theor Biol 363:53-61. doi:10.1016/ j.jtbi.2014.07.033

Lazdunski AM, Ventre I, Sturgis JN (2004) Regulatory circuits and communication in Gram-negative bacteria. Nat Rev Microbiol 2:581-592

Lee J, Jayaraman A, Wood TK (2007) Indole is an inter-species biofilm signal mediated by SdiA. Microbiol 7:42. doi:10.1186/1471-2180-7-42

Lesic B, de Lorenzo V, Lepine F, Deziel E, Zhang J, Zhang Q, Padfield K (2007) Inhibitors of pathogen intercellular signals as selective anti-infective compounds. PLoS Pathog 3:1229-1239

Li J, Wang L, Hashimoto Y, Tsao CY, Wood TK, Valdes JJ, Zafiriou E, Bentley WE (2006) A stochastic model of Escherichia coli ai-2 quorum signal circuit reveals alternative synthesis pathways. Mol Syst Biol 2. doi: $10.1038 / \mathrm{msb} 4100107$

Liu X, Zhou P, Wang R (2012) Switch-like regulation of signal transduction by small RNA-mediated quorum sensing. In: 2012 IEEE 6th international conference on systems biology (ISB), pp 164-168. doi:10. 1109/ISB.2012.6314130

Liu X, Zhou P, Wang R (2013) Small rna-mediated switch-like regulation in bacterial quorum sensing. IET Syst Biol 7:182-187. doi:10.1049/iet-syb.2012.0059

Lyon GJ, Novick RP (2004) Peptide signaling in Staphylococcus aureus and other Gram-positive bacteria. Peptides 25:1389-1403

Maeda T, García-Contreras R, Pu M, Sheng L, García LR, Tomas M, Wood TK (2012) Quorum quenching quandary: resistance to antivirulence compounds. ISME J 6:493-501. doi:10.1038/ismej.2011.122

Majumdar S, Datta S, Roy S (2012) Mathematical modelling of quorum sensing and bioluminescence in bacteria. IJAAS 1(3):139-146

McMillen D, Kopell N, Hasty J, Collins JJ (2002) Synchronizing genetic relaxation oscillators by intercell signaling. Proc Natl Acad Sci USA 99:679-684. doi:10.1073/pnas.022642299

Mehra S, Charaniya S, Takano E, Hu WS (2008) A bistable gene switch for antibiotic biosynthesis: the butyrolactone regulon in Streptomyces coelicolor. PLoS One 3:e2724. doi:10.1371/journal.pone.0002724

Mehta P, Goyal S, Long T, Bassler BL, Wingreen NS (2009) Information processing and signal integration in bacterial quorum sensing. Mol Syst Biol 5. doi:10.1038/msb.2009.79

Melke P, Sahlin P, Levchenko A, Jönsson H (2010) A cell-based model for quorum sensing in heterogeneous bacterial colonies. PLoS Comput Biol 6(6):e1000819

Megerle JA, Fritz G, Gerland U, Jung K, Rädler JO (2008) Timing and dynamics of single cell gene expression in the Arabinose utilization system. Biophys J 95: 2103-2115. URL: http://linkinghub. elsevier.com/retrieve/pii/S0006349508701681

Meyer A, Megerle JA, Kuttler C, Müller J, Aguilar C, Eberl L, Hense BA, Rädler JO (2012) Dynamics of ahl mediated quorum sensing under flow and non-flow conditions. Phys Biol 9: 026007. URL: http:// stacks.iop.org/1478-3975/9/i=2/a=026007

Miller MB, Bassler BL (2001) Quorum sensing in bacteria. Annu Rev Microbiol 55:165-199. doi:10.1146/ annurev.micro.55.1.165

Müller J, Kuttler C, Hense BA (2008a) Sensitivity of the quorum sensing system is achieved by low pass filtering. Biosystems 92:76-81. doi:10.1016/j.biosystems.2007.12.004

Müller J, Kuttler C, Hense BA, Rothballer M, Hartmann A (2006) Cell-cell communication by quorum sensing and dimension-reduction. J Math Biol 53:672-702. doi:10.1007/s00285-006-0024-Z 
Müller J, Kuttler C, Hense BA, Zeiser S, Liebscher V (2008) Transcription, intercellular variability and correlated random walk. Math Biosci 216:30-39. doi:10.1016/j.mbs.2008.08.003

Müller J, Uecker H (2013) Approximating the dynamics of communicating cells in a diffusive medium by odeshomogenization with localization. J Math Biol 67:1023-1065. doi:10.1007/s00285-012-0569-y

Nadell CD, Xavier JB, Levin SA, Foster KR (2008) The evolution of quorum sensing in bacterial biofilms. PLoS Biol 6:e14. doi:10.1371/journal.pbio.0060014

Nealson KH, Hastings JW (1979) Bacterial bioluminescence: its control and ecological significance. Microbiol Rev 43:496-518. URL: http://mmbr.asm.org/content/43/4/496.short

Netotea S, Bertani I, Steindler L, Kerényi Á, Venturi V, Pongor S (2009) A simple model for the early events of quorum sensing in Pseudomonas aeruginosa: modeling bacterial swarming as the movement of an “activation zone". Biol Direct 4:6-6. doi:10.1186/1745-6150-4-6

Nilsson P, Olofsson A, Fagerlind M, Fagerström T, Rice S, Kjelleberg S, Steinberg P (2001) Kinetics of the ahl regulatory system in a model biofilm system: how many bacteria constitute a "quorum"? J Mol Biol 309(3):631-640. doi:10.1006/jmbi.2001.4697

Ofria C, Wilke CO (2004) Avida: A software platform for research in computational evolutionary biology. Artif Life 10:191-229. doi:10.1162/106454604773563612

O’Loughlin CT, Miller LC, Siryaporn A, Drescher K, Semmelhack MF, Bassler BL (2013) A quorumsensing inhibitor blocks Pseudomonas aeruginosa virulence and biofilm formation. Proc Natl Acad Sci USA 110:17981-17986

Palmer KL, Aye LM, Whiteley M (2007) Nutritional cues control Pseudomonas aeruginosa multicellular behavior in cystic fibrosis sputum. J Bacteriol 189:8079-8087

Perez P, Weiss J, Hagen S (2011) Noise and crosstalk in two quorum-sensing inputs of Vibrio fischeri. BMC Syst Biol 5:153. doi:10.1186/1752-0509-5-153

Pérez-Jiménez MJ, Romero-Campero FJ (2006) P systems, a new computational modelling tool for systems biology. In: Priami C, Plotkin G (eds) Transactions on computational systems biology VI, vol. 4220 of Lecture Notes in Computer Science, Springer, Berlin, pp 176-197. doi:10.1007/11880646_8

Pérez-Velázquez J, Quiñones B, Hense BA, Kuttler C (2015) A mathematical model to investigate quorum sensing regulation and its heterogeneity in Pseudomonas syringae on leaves. Ecol Complex 21:128141. doi:10.1016/j.ecocom.2014.12.003

Picioreanu C, Kreft JU, van Loosdrecht MCM (2004) Particle-based multidimensional multispecies biofilm model. Appl Environ Microbiol 70:3024-3040. doi:10.1128/AEM.70.5.3024-3040.2004

Platt TG, Fuqua C (2010) What's in a name? The semantics of quorum sensing. Trends Microbiol 18:383387. doi:10.1016/j.tim.2010.05.003

Popat R, Crusz SA, Messina M, Williams P, West SA, Diggle SP (2012) Quorum-sensing and cheating in bacterial biofilms. Proc R Soc B 279:4765-4771. doi:10.1098/rspb.2012.1976

Pradhan BB, Chatterjee S (2014) Reversible non-genetic phenotypic heterogeneity in bacterial quorum sensing. Mol Microbiol 22:557-569. doi:10.1111/mmi.12575

Queller DC (1992) Quantitative genetics, inclusive fitness, and group selection. Am Nat pp 540-558

Redfield RJ (2002) Is quorum sensing a side effect of diffusion sensing? Trends Microbiol 10:365-370. doi:10.1016/S0966-842X(02)02400-9

Romero-Campero FJ, Pérez-Jiménez MJ (2008) A model of the quorum sensing system in Vibrio fischeri using p systems. Artif Life 14:95-109. doi:10.1162/artl.2008.14.1.95

Rumbaugh KP, Diggle SP, Watters CM, Ross-Gillespie A, Griffin AS, West SA (2009) Quorum sensing and the social evolution of bacterial virulence. Curr Biol 19:341-345. doi:10.1016/j.cub.2009.01.050

Schaadt N, Steinbach A, Hartmann R, Helms V (2013) Rule-based regulatory and metabolic model for quorum sensing in P. aeruginosa. BMC Syst Biol 7:81. doi:10.1186/1752-0509-7-81

Sepulchre JA, Reverchon S, Nasser W (2007) Modeling the onset of virulence in a pectinolytic bacterium. J Theor Biol 244:239-257. doi:10.1016/j.jtbi.2006.08.010

Smith R, Coast J (2013) The true cost of antimicrobial resistance. BMJ 346. doi:10.1136/bmj.f1493

Stacy AR, Diggle SP, Whiteley M (2012) Rules of engagement: defining bacterial communication. Curr Opin Microbiol 15:155-161. doi:10.1016/j.mib.2011.11.007

Taga ME, Bassler BL (2003) Chemical communication among bacteria. Proc Natl Acad Sci USA 100:14549-14554. doi:10.1073/pnas.1934514100

Tang W, Wu Q, Saunders J (2006) A novel model for bacterial foraging in varying environments. In: Gavrilova M, Gervasi O, Kumar V, Tan C, Taniar D, Laganá A, Mun Y, Choo H (eds) Computational science and its applications-ICCSA 2006, vol. 3980 of Lecture Notes in Computer Science, Springer, Berlin, pp 556-565 
Tang W, Wu Q, Saunders J (2007) Individual-based modeling of bacterial foraging with quorum sensing in a time-varying environment. In: Marchiori E, Moore J, Rajapakse J (eds) Evolutionary computation,machine learning and data mining in bioinformatics, vol. 4447 of Lecture Notes in Computer Science, Springer, Berlin, pp 280-290. doi:10.1007/978-3-540-71783-6_27

Tanouchi Y, Tu D, Kim J, You L (2008) Noise reduction by diffusional dissipation in a minimal quorum sensing motif. PLoS Comput Biol 4:e1000167. doi:10.1371/journal.pcbi.1000167

Teng SW, Wang Y, Tu KC, Long T, Mehta P, Wingreen NS, Bassler BL, Ong N (2010) Measurement of the copy number of the master quorum-sensing regulator of a bacterial cell. Biophys J 98:2024-2031. doi:10.1016/j.bpj.2010.01.031

Tu KC, Long T, Svenningsen SL, Wingreen NS, Bassler BL (2010) Negative feedback loops involving small regulatory RNAs precisely control the vibrio harveyi quorum-sensing response. Mol Cell 37:567-579. doi:10.1016/j.molcel.2010.01.022

Uecker H, Müller J, Hense B (2014) Individual-based model for quorum sensing with background flow. Bull Math Biol 76:1727-1746. doi:10.1007/s11538-014-9974-2

Ullner E, Zaikin A, Volkov EI, García-Ojalvo J (2007) Multistability and clustering in a population of synthetic genetic oscillators via phase-repulsive cell-to-cell communication. Phys Rev Lett 99:148103. doi:10.1103/PhysRevLett.99.148103

Uroz S, Chhabra SR, Cámara M, Williams P, Oger P, Dessaux Y (2005) N-acylhomoserine lactone quorumsensing molecules are modified and degraded by rhodococcus erythropolis W2 by both amidolytic and novel oxidoreductase activities. Microbiology 151(10):3313-3322. doi:10.1099/mic.0.27961-0

Vainstein V, Kirnasovsky OU, Kogan Y, Agur Z (2012) Strategies for cancer stem cell elimination: Insights from mathematical modeling. J Theor Biol 298:32-41. doi:10.1016/j.jtbi.2011.12.016

van Gestel J, Nowak MA, Tarnita CE (2012) The evolution of cell-to-cell communication in a sporulating bacterium. PLoS Comput Biol 8:e1002818. doi:10.1371/journal.pcbi.1002818

Vaughan B, Smith B, Chopp DL (2010) The influence of fluid flow on modeling quorum sensing in bacterial biofilms. Bull Math Biol 72(5):1143-1165. doi:10.1007/s11538-009-9485-8

Viretta AU, Fussenegger M (2004) Modeling the quorum sensing regulatory network of human-pathogenic Pseudomonas aeruginosa. Biotechnol Prog 20:670-678. doi:10.1021/bp0343231

Wang M, Schaefer AL, Dandekar AA, Greenberg EP (2015) Quorum sensing and policing of Pseudomonas aeruginosa social cheaters. Proc Natl Acad Sci USA 112:2187-2191. doi:10.1073/pnas.1500704112

Ward J (2008) Mathematical modeling of quorum-sensing control in biofilms. In: Balaban N (ed) Control of biofilm infections by signal manipulation, vol. 2 of Springer Series on Biofilms. Springer, Berlin, pp 79-108

Ward J, King J (2012) Thin-film modelling of biofilm growth and quorum sensing. J Eng Math 73:71-92. doi:10.1007/s10665-011-9490-4

Ward JP, King JR, Koerber AJ, Croft JM, Sockett RE, Williams P (2003) Early development and quorum sensing in bacterial biofilms. J Math Biol 47:23-55. doi:10.1007/s00285-002-0190-6

Ward JP, King JR, Koerber AJ, Croft JM, Sockett RE, Williams P (2004) Cell-signalling repression in bacterial quorum sensing. Math Med Biol 21:169-204. doi:10.1093/imammb/21.3.169

Ward JP, King JR, Koerber AJ, Williams P, Croft JM, Sockett RE (2001) Mathematical modelling of quorum sensing in bacteria. Math Med Biol 18:263-292. doi:10.1093/imammb/18.3.263

Waters CM, Bassler BL (2005) Quorum sensing: cell-to-cell communication in bacteria. Annu Rev Cell Dev Biol 21:319-346. doi:10.1146/annurev.cellbio.21.012704.131001

Weber M, Buceta J (2013) Dynamics of the quorum sensing switch: stochastic and non-stationary effects. BMC Syst Biol 7:6. doi:10.1186/1752-0509-7-6

West SA, Griffin AS, Gardner A (2007) Evolutionary explanations for cooperation. Curr Biol 17:R661R672

West SA, Winzer K, Gardner A, Diggle S (2012) Quorum sensing and the confusion about diffusion. Trends Microbiol 20:586-594. doi:10.1016/j.tim.2012.09.004

Whitaker RD, Pember S, Wallace BC, Brodley CE, Walt DR (2011) Single cell time-resolved quorum responses reveal dependence on cell density and configuration. J Biol Chem 286:21623-21632. URL: http://www.jbc.org/content/286/24/21623.abstract

Wilder CN, Allada G, Schuster M (2009) Instantaneous within-patient diversity of Pseudomonas aeruginosa quorum-sensing populations from cystic fibrosis lung infections. Infect Immun 77:5631-5639

Williams JW, Cui X, Levchenko A, Stevens AM (2008) Robust and sensitive control of a quorum-sensing circuit by two interlocked feedback loops. Mol Syst Biol 4:234-234. doi:10.1038/msb.2008.70 
Winzer K, Hardie KR, Williams P (2002) Bacterial cell-to-cell communication: sorry, can't talk now gone to lunch!. Curr Opin Microbiol 5:216-222. doi:10.1016/S1369-5274(02)00304-1

Wynendaele E, Bronselaer A, Nielandt J, D’Hondt M, Stalmans S, Bracke N, Verbeke F, Van De Wiele C, De Tré G, De Spiegeleer B (2013) Quorumpeps database: chemical space, microbial origin and functionality of quorum sensing peptides. Nucleic Acids Res 41:655-659. doi:10.1093/nar/gks1137

You L, Cox RS, Weiss R, Arnold FH (2004) Programmed population control by cell-cell communication and regulated killing. Nature 428:868-871. doi:10.1038/nature02491

Zhou T, Zhang J, Yuan Z, Chen L (2008) Synchronization of genetic oscillators. Chaos 18. doi:10.1063/1. 2978183

Zhu J, Miller MB, Vance RE, Dziejman M, Bassler BL, Mekalanos JJ (2002) Quorum-sensing regulators control virulence gene expression in Vibrio cholerae. Proc Natl Acad Sci 99:3129-3134 